\title{
A Novel Method of Ranking Intuitionistic Fuzzy Numbers using Value and $\theta$ Multiple of Ambiguity at Flexibility Parameters
}

Rituparna Chutia ( $\sim$ Rituparnachutia7@gmail.com )

Cotton University https://orcid.org/0000-0002-5323-3698

\section{Research Article}

Keywords: Intuitionistic fuzzy number, value, ambiguity, flexibility parameter

Posted Date: June 7th, 2021

DOI: https://doi.org/10.21203/rs.3.rs-557307/v1

License: (c) (i) This work is licensed under a Creative Commons Attribution 4.0 International License.

Read Full License

Version of Record: A version of this preprint was published at Soft Computing on August 14th, 2021. See the published version at https://doi.org/10.1007/s00500-021-06102-8. 


\title{
A novel method of ranking intuitionistic fuzzy numbers using value and $\theta$ multiple of ambiguity at flexibility
} parameters

\author{
Rituparna Chutia * \\ Department of Mathematics, Cotton University, Guwahati, Assam 781001, India.
}

\begin{abstract}
In this paper a novel method of ordering intuitionistic fuzzy numbers, based on the notions of 'value' and $\theta$-multiple of 'ambiguity' of an intuitionistic fuzzy number, is developed. Further, the flexibility parameters, of decision-making at $(\alpha, \beta)$-levels, are used in the method. These parameters allow the decision-maker to take decisions at various $(\alpha, \beta)$-levels of decision-making. Many a times, all the reasonable properties of ranking intuitionistic fuzzy numbers were never checked in the existing studies. However, in this study an utmost attempt is being made to study the reasonable properties thoroughly. Further, the existing methods are mostly based on intuition and the geometry of the intuitionistic fuzzy numbers. However, the proposed method completely complies with the reasonable properties of ranking intuitionistic fuzzy numbers as well as the coherent intuition and the geometry of the intuitionistic fuzzy numbers. Further, newer properties are also being developed in this study. These prove the novelty of the proposed method. Further, a few numerical examples are discussed that demonstrates the proposed method.
\end{abstract}

Keywords. Intuitionistic fuzzy number, value, ambiguity, flexibility parameter.

\section{Introduction}

The information involved in problems of decision-making, operation research, risk analysis and so forth may be vague, in such situations fuzzy set theory is very essential. Most of the times such vague information are often expressed as linguistic terms which in turn can be expressed as fuzzy numbers. Hence, in a decision-making process the ordering of fuzzy number is essential for making a proper decision. The concept of ordering fuzzy quantities is possible due to the idea of ranking fuzzy numbers. This situation of ordering fuzzy quantities was first overcome by the pioneer works, through developing a method using maximizing set, of Jain [17, 18] and Dubois and Prade [14].

Atanassov [1] in 1986 proposed the concept of intuitionistic fuzzy sets to cover the absence or incomplete information in a fuzzy set. The intuitionistic fuzzy set (IFS) is the generalization of fuzzy set adding the concept of non-membership function in fuzzy set. This generalization in fuzzy sets to IFSs adds more abundant and flexible information as compared to fuzzy sets. Atanassov [2, 3, 5] are the some of the works that have abundant concepts and pioneering

\footnotetext{
${ }^{*}$ E-mail address : rituparnachutia7@gmail.com
} 
works on IFS. Li [20] initiated the concept of intuitionistic fuzzy number (IFN) as an ill-known quantity that holds information about the membership and non-membership of an element simultaneously. As such, its application has been evident in the numerous field of decisionmaking and risk analysis and optimization, etc. Generally, in these problems selection among the alternatives is an indispensable task. Hence, ordering of the IFNs is very essential for an a proper and approximate decision.

For years, the ordering of IFNs got little attention among the researchers as compared to the ordering of fuzzy numbers. However, in recent times ordering of IFNs are gearing up, as such several definitions of ordering IFNs are being forwarded. Further, most of the existing methods are based on the geometry and the coherent intuition, and a little attention is drawn towards fulfilling all the reasonable properties of ranking IFNs. Some of the works on ranking IFNs are by Das \& Guha [10, Grzegorzewski [16], Kumar \& Kaur [19], Mitchell [22], Nehi [26], Salahshour et al. [28, Seikh et al. [29], Wan \& Li [31], Zhang \& Xu [37] and Chutia \& Saikia [7]. Here, a brief review, of a few existing methods, of ordering IFNs is as follows. Interpreting each IFN to an ensemble of ordinary fuzzy numbers, a statistical viewpoint method was developed by Mitchell [22] for ranking IFNs. Wang and Zhang [32] transformed the ranking IFNs into the ranking of interval numbers and used in ranking the IFNs. The method seems not popular among the researcher as the resulting index is interval numbers. Value and ambiguity were found to incorporate in ranking IFNs in several attempts by Das \& De [9], Dubey \& Mehra [13], Zeng et al. [36] and many more. Nayagam [25] performed a thorough review of some of the existing methods of ranking IFNs, pointing out the limitations of various methods, and developed a new improved value index of ranking IFNs. However, the images of IFNs and the reasonable properties were never incorporated in this study. Further, the ratio of the value index with the ambiguity index, to rank triangular IFNs, was developed by Li [21]; and applied to multi-attribute decision-making problems. Later, the limitations and of Li's approach, due to non linearity, was removed by by De and Das [11]. Nan et al. [23] used membership function average indexes to rank IFNs. The notion of minimum possibility variance coefficients, in ranking IFNs, was introduced by Wan [30]. Rezvani [27] again used values and ambiguities of the membership degree and the non-membership degree of ordering IFNs and defined the value-index and the ambiguity-index. Nayagam et al. [24] defined eight different classes of IFNs and defined different ranking indexes for these classes. In a recent study, Chutia \& Saikia [7] studied the ordering of IFNs based on the concept of 'value' and 'ambiguity' calculated at various $(\alpha, \beta)$-levels of decision-making. However, limitations in ordering the images of IFNs when 'values' are equal is mentioned in the same. Equivalently, Dutta \& Saikia [15] developed a method of ranking IFNs, based on the notion of 'value' multiplied with mean and 'ambiguity' multiplied with mean through the index of optimism, following Chutia \& Siakia [7]. However, the images of the IFNs were not incorporated in this study. Further, the reasonable properties of ordering IFNs were also not verified. Very recently, Darehmiraki [8] introduced an ordering method of IFNs through the concept of areas calculated on the left side at various $(\alpha, \beta)$-levels of decision-making.

The above literature review admits that the existing studies rarely ranks the images and reasonable properties of ranking IFNs. Hence, in this study, these points will be incorporated. Hence, in this current study the objectives are as follows. The main objective of this work is to develop a method of ordering IFNs. Generally, there exists numerous method of ordering IFNs. Most the existing methods, generally, consider the coherent intuition and the geometry of the IFNs to validate the method. Only in a few cases, the reasonable properties of Wang and Kerre [33] are considered as base properties to validate the method. In this paper, a novel method of ordering IFNs, considering the notions of 'value' and $\theta$-multiple of 'ambiguity', is developed. The 'flexibility parameter' allows the decision-makers to take decision at levels $(\alpha, \beta)$ of the IFNs. The method overcome all the limitations of the existing methods. Further, 
newer properties are also suggested in this work. One of the interesting features of the present method is that it successfully and consistently rank the IFNs and their corresponding images, which was never attempted in any of the existing study. Apart from this, the method also ranks IFNs that are symmetric about the $y$-axis.

Apart from this section, the rest of the paper is organized as follows. Section 2 introduces the basic definitions of IFN and related definitions essential for the discussion. Section 3 discusses the definitions and theorems of the proposed ranking method. Further, the rationality validation of the proposed raking method is discussed by proving the Wang and Kerre's [33] reasonable properties on ranking fuzzy quantities. Apart from that, newer properties are also discussed. Section 4 some numerical examples are discussed that highlights some of the main features of the proposed method. Finally, in section 5 conclusions and the main features of the proposed method are highlighted.

\section{Preliminaries}

In this section, a brief review of related definitions and notations of IFN is being discussed which are essential for further study.

Definition 2.1. 24] An IFN $\widetilde{a}=\left\langle\mu_{\tilde{a}}, \nu_{\tilde{a}}\right\rangle$ in the set of real numbers $\mathbb{R}$ with membership function and non-membership functions are defined as

$$
\mu_{\tilde{a}}(x)= \begin{cases}f_{\widetilde{a}}(x), & \text { if } a \leq x \leq x_{0}, \\ 1, & \text { if } x_{0} \leq x \leq y_{0}, \\ g_{\widetilde{a}}(x), & \text { if } y_{0} \leq x \leq b, \\ 0, & \text { otherwise }\end{cases}
$$

and

$$
\nu_{\tilde{a}}(x)= \begin{cases}h_{\widetilde{a}}(x), & \text { if } c \leq x \leq x_{0}, \\ 0, & \text { if } x_{0} \leq x \leq y_{0} \\ k_{\widetilde{a}}(x), & \text { if } y_{0} \leq x \leq d, \\ 1, & \text { otherwise, }\end{cases}
$$

respectively, where $0 \leq \mu_{\widetilde{a}}(x)+\nu_{\tilde{a}}(x) \leq 1$ and $c, a, x_{0}, y_{0}, b, d \in \mathbb{R}$ such that $c \leq a, x_{0} \leq y_{0}$, $b \leq d$ and the functions $f_{\tilde{a}}, g_{\tilde{a}}, h_{\tilde{a}}, k_{\tilde{a}}: \mathbb{R} \longrightarrow[0,1]$ are the legs of membership function $\mu_{\tilde{a}}$ and non-membership function $\nu_{\tilde{a}}$. The functions $f_{\tilde{a}}$ and $k_{\tilde{a}}$ are non-decreasing continuous functions and the functions $h_{\widetilde{a}}$ and $g_{\widetilde{a}}$ are non-increasing continuous functions. Hence, the IFN can also be denoted by $\widetilde{a}=\left\langle\left(a, x_{0}, y_{0}, b\right),\left(c, x_{0}, y_{0}, d\right)\right\rangle$.

Definition 2.2. 24] Let $\widetilde{a}=\left\langle\left(a, x_{0}, y_{0}, b\right),\left(c, x_{0}, y_{0}, d\right)\right\rangle$ be a TrIFN where the real numbers are such that $c \leq a, x_{0} \leq y_{0}, b \leq d$, then the membership function and the non-membership function are defined as

$$
\mu_{\tilde{a}}(x)= \begin{cases}\frac{x-a}{x_{0}-a} & \text { if } a \leq x \leq x_{0} \\ 1 & \text { if } x_{0} \leq x \leq y_{0} \\ \frac{b-x}{b-y_{0}} & \text { if } y_{0} \leq x \leq b \\ 0 & \text { otherwise }\end{cases}
$$


and

$$
\nu_{\widetilde{a}}(x)= \begin{cases}\frac{x-x_{0}}{c-x_{0}} & \text { if } c \leq x \leq x_{0} \\ 0 & \text { if } x_{0} \leq x \leq y_{0} \\ \frac{x-y_{0}}{d-y_{0}} & \text { if } y_{0} \leq x \leq d \\ 1 & \text { otherwise }\end{cases}
$$

respectively, where $0 \leq \mu_{\widetilde{a}}(x)+\nu_{\widetilde{a}}(x) \leq 1$ and $\mu_{\widetilde{a}}(x)$ denotes the degree of belongingness of an element and $\nu_{\widetilde{a}}(x)$ denotes the degree of non-belongingness of an element in $\widetilde{a}$.

Definition 2.3. [21] The IF index of an element $x$ in $\widetilde{a}$ is the degree of the indeterminacy membership $\pi_{\widetilde{a}}$ of the element $x$ in $\widetilde{a}$ is defined as

$$
\pi_{\widetilde{a}}(x)=1-\mu_{\widetilde{a}}(x)-\nu_{\widetilde{a}}(x) .
$$

As like the fuzzy number, the cut sets of IFN can also be defined for the membership and the non-membership functions. These sets are defined by Atanassov [4, 21] as follows:

A $(\alpha, \beta)$-cut set, of a IFN $\widetilde{a}$, is a crisp subset of $\mathbb{R}$, which is defined as $\widetilde{a}_{\alpha, \beta}=\left\{x \mid \mu_{\widetilde{a}}(x) \geq\right.$ $\left.\alpha, \nu_{\tilde{a}}(x) \leq \beta\right\}$, where $0 \leq \alpha+\beta \leq 1, \mu_{\tilde{a}}$ and $\nu_{\tilde{a}}$ are the membership and the non-membership function of $\widetilde{a}$ respectively. A $\alpha$-cut set, of a IFN $\widetilde{a}$, is a crisp subset of $\mathbb{R}$, which is defined as $\widetilde{a}_{\alpha}=\left\{x \mid \mu_{\widetilde{a}}(x) \geq \alpha\right\}$ where $0 \leq \alpha \leq 1, \mu_{\widetilde{a}}$ is the membership function of $\widetilde{a}$. It is clear that $\widetilde{a}_{\alpha}$ represents a closed interval, denoted by $\widetilde{a}_{\alpha}=\left[L_{\widetilde{a}}^{\mu}(\alpha), R_{\widetilde{a}}^{\mu}(\alpha)\right]$, which can be calculated using the membership function Eq. 3 as

$$
\widetilde{a}_{\alpha}=\left[L_{\widetilde{a}}^{\mu}(\alpha), R_{\widetilde{a}}^{\mu}(\alpha)\right]=\left[a+\alpha\left(x_{0}-a\right), b-\alpha\left(b-y_{0}\right)\right] .
$$

A $\beta$-cut set of a IFN $\widetilde{a}$ is a crisp subset of $\mathbb{R}$, which is defined as $\widetilde{a}_{\beta}=\left\{x \mid \nu_{\widetilde{a}}(x) \leq \beta\right\}$, where $0 \leq \beta \leq 1, \nu_{\tilde{a}}$ is the non-membership function of $\widetilde{a}$. It is clear that $\widetilde{a}_{\beta}$ represents a closed interval, denoted by $\widetilde{a}_{\beta}=\left[L_{\widetilde{a}}^{\nu}(\beta), R_{\widetilde{a}}^{\nu}(\beta)\right]$, which can be calculated using the non-membership function Eq. 4 as

$$
\widetilde{a}_{\beta}=\left[L_{\widetilde{a}}^{\nu}(\beta), R_{\widetilde{a}}^{\nu}(\beta)\right]=\left[x_{0}+\beta\left(c-x_{0}\right), y_{0}+\beta\left(d-y_{0}\right)\right] .
$$

The notation fuzzy centre of the membership and non-membership function are defined as

$$
C_{\widetilde{a}}^{\mu}(\alpha)=\frac{L_{\widetilde{a}}^{\mu}(\alpha)+R_{\widetilde{a}}^{\mu}(\alpha)}{2} \text { and } C_{\widetilde{a}}^{\nu}(\beta)=\frac{L_{\widetilde{a}}^{\nu}(\beta)+R_{\widetilde{a}}^{\nu}(\beta)}{2}
$$

for all $0 \leq \alpha \leq 1$ and $0 \leq \beta \leq 1$.

The support of a IFN $\widetilde{a}$ for the membership function is defined as $\operatorname{supp}\left(\mu_{\widetilde{a}}\right)=\left\{x \mid \mu_{\widetilde{a}}(x) \geq\right.$ $0\}$. and the support of the non-membership function is defined as $\operatorname{supp}\left(\nu_{\tilde{a}}\right)=\left\{x \mid \nu_{\tilde{a}}(x) \leq\right.$ $1\}$. Further, the following notation are used in this study, $L_{\widetilde{a}}^{\mu}(0)=\inf \operatorname{supp}\left(\mu_{\tilde{a}}\right), R_{\widetilde{a}}^{\mu}(0)=$ $\sup \operatorname{supp}\left(\mu_{\widetilde{a}}\right), L_{\widetilde{a}}^{\nu}(1)=\inf \operatorname{supp}\left(\nu_{\tilde{a}}\right), R_{\widetilde{a}}^{\nu}(1)=\sup \operatorname{supp}\left(\nu_{\tilde{a}}\right)$.

The image of an IFN $\widetilde{a}=\left\langle\mu_{\widetilde{a}}, \nu_{\tilde{a}}\right\rangle$ is an IFN given by $-\widetilde{a}=\left\langle-\mu_{\widetilde{a}},-\nu_{\tilde{a}}\right\rangle$. Thus if $\widetilde{a}_{\alpha}=$ $\left[L_{\widetilde{a}}^{\mu}(\alpha), R_{\widetilde{a}}^{\mu}(\alpha)\right]$ and $\widetilde{a}_{\beta}=\left[L_{\widetilde{a}}^{\nu}(\beta), R_{\widetilde{a}}^{\nu}(\beta)\right]$ be the $\alpha$-cut and $\beta$-cut sets of $\widetilde{a}$, then the $\alpha$-cut and $\beta$-cut sets of $-\widetilde{a}$ are $-\widetilde{a}_{\alpha}=\left[-R_{\widetilde{a}}^{\mu}(\alpha),-L_{\widetilde{a}}^{\nu}(\alpha)\right]$ and $-\widetilde{a}_{\beta}=\left[-R_{\widetilde{a}}^{\nu}(\beta),-L_{\widetilde{a}}^{\nu}(\beta)\right]$ respectively. An IFN $\widetilde{a}$ is called symmetric about $y$-axis if, $-L_{\widetilde{a}}^{\mu}(\alpha)=R_{\widetilde{a}}^{\mu}(\alpha)$ and $-L_{\widetilde{a}}^{\nu}(\beta)=R_{\widetilde{a}}^{\nu}(\beta)$. Further, an IFN $\widetilde{a}$ is called an crisp-valued IFN, if $\widetilde{a}=\langle a, a\rangle$, that is, $\widetilde{a}=a \in \mathbb{R}$.

The arithmetic operations of IFN are defined by Li [20, 21] while developing a methodology of ranking IFNs. Further, it arithmetic operations are extensively studied by Chakraborty et al. [6] using different methodology, namely, $(\alpha, \beta)$-cut method, vertex method and extension principle method. The $(\alpha, \beta)$-cut method of arithmetic operations are adopted in this research 
work. Let $\widetilde{a}=\left\langle\left(a, x_{0}, y_{0}, b\right),\left(c, x_{0}, y_{0}, d\right)\right\rangle$ and $\widetilde{b}=\left\langle\left(p, m_{0}, n_{0}, q\right),\left(r, m_{0}, n_{0}, s\right)\right\rangle$ be two IFNs and $\lambda \in \mathbb{R}-\{0\}$. Let the $\alpha$-cut and $\beta$-cut sets of $\widetilde{a}$ and $\widetilde{b}$ be $\widetilde{a}_{\alpha}=\left[L_{\widetilde{a}}^{\mu}(\alpha), R_{\widetilde{a}}^{\mu}(\alpha)\right], \widetilde{a}_{\beta}=\left[L_{\widetilde{a}}^{\nu}(\beta), R_{\widetilde{a}}^{\nu}(\beta)\right]$ and $\widetilde{b}_{\alpha}=\left[L_{\tilde{b}}^{\mu}(\alpha), R_{\tilde{b}}^{\mu}(\alpha)\right], \widetilde{b}_{\beta}=\left[L_{\tilde{b}}^{\nu}(\beta), R_{\widetilde{b}}^{\nu}(\beta)\right]$ respectively, then the arithmetic operations are defined as

$$
\begin{aligned}
& {[\widetilde{a}+\widetilde{b}]_{\alpha}=\left[L_{\widetilde{a}}^{\mu}(\alpha)+L_{\widetilde{b}}^{\mu}(\alpha), R_{\widetilde{a}}^{\mu}(\alpha)+R_{\tilde{b}}^{\mu}(\alpha)\right],[\widetilde{a}+\widetilde{b}]_{\beta}=\left[L_{\widetilde{a}}^{\nu}(\beta)+L_{\widetilde{b}}^{\nu}(\beta), R_{\widetilde{a}}^{\nu}(\beta)+R_{\widetilde{b}}^{\nu}(\beta)\right] ;} \\
& {[\widetilde{a}-\widetilde{b}]_{\alpha}=\left[L_{\widetilde{a}}^{\mu}(\alpha)-R_{\widetilde{b}}^{\mu}(\alpha), R_{\widetilde{a}}^{\mu}(\alpha)-R_{\widetilde{b}}^{\mu}(\alpha)\right],[\widetilde{a}-\widetilde{b}]_{\beta}=\left[L_{\widetilde{a}}^{\nu}(\beta)-R_{\widetilde{b}}^{\nu}(\beta), R_{\widetilde{a}}^{\nu}(\beta)-L_{\widetilde{b}}^{\nu}(\beta)\right] ;} \\
& {[\lambda \widetilde{a}]_{\alpha}=\left\{\begin{array}{lll}
{\left[\lambda L_{\widetilde{a}}^{\mu}(\alpha), \lambda R_{\tilde{a}}^{\mu}(\alpha)\right]} & \text { if } \lambda>0, \\
{\left[\lambda R_{\widetilde{a}}^{\mu}(\alpha), \lambda L_{\widetilde{a}}^{\mu}(\alpha)\right]} & \text { if } \lambda<0,
\end{array},[\lambda \widetilde{a}]_{\beta}= \begin{cases}{\left[\lambda L_{\widetilde{a}}^{\nu}(\beta), \lambda R_{\tilde{a}}^{\nu}(\beta)\right]} & \text { if } \lambda>0, \\
{\left[\lambda R_{\widetilde{a}}^{\nu}(\beta), \lambda L_{\widetilde{a}}^{\nu}(\beta)\right]} & \text { if } \lambda<0,\end{cases} \right.}
\end{aligned}
$$

Eventually, these arithmetic operations on the $(\alpha, \beta)$-cut is calcuated to obtained the following expressions, which were clearly described by Chakraborty et al. [6].

$$
\begin{aligned}
& \widetilde{a}+\widetilde{b}=\left\langle\left(a+p, x_{0}+m_{0}, y_{0}+n_{0}, b+q\right),\left(c+r, x_{0}+m_{0}, y_{0}+n_{0}, d+s\right)\right\rangle \\
& \widetilde{a}-\widetilde{b}=\left\langle\left(a-q, x_{0}-n_{0}, y_{0}-m_{0}, b-p\right),\left(c-s, x_{0}-n_{0}, y_{0}-m_{0}, d-r\right)\right\rangle \\
& \lambda \widetilde{a}= \begin{cases}\left\langle\left(\lambda a, \lambda x_{0}, \lambda y_{0}, \lambda b\right),\left(\lambda c, \lambda x_{0}, \lambda y_{0}, \lambda d\right)\right\rangle & \text { if } \lambda>0, \\
\left\langle\left(\lambda b, \lambda y_{0}, \lambda x_{0}, \lambda a\right),\left(\lambda d, \lambda y_{0}, \lambda x_{0}, \lambda c\right)\right\rangle & \text { if } \lambda<0,\end{cases}
\end{aligned}
$$

The collection of the IFNs that follows the above defined arithmetic operations with bounded support and convex IFNs are denoted by the set $\mathcal{I F}$.

\section{$3 \quad$ The proposed method of ranking IFN}

The notions of value and ambiguity of a fuzzy number were developed by Delgado et al. [12] which basically represent and capture the information of a fuzzy number. Later, these quantities were widely used in ranking fuzzy numbers in various studies. Hence, in this study, these quantities are used, but from a different prospective.

\subsection{Definitions and notions related to the proposed method}

In this subsection, the basic definitions that are essential to develop the current method is forwarded. Further, a few propositions are also forwarded which basically helps in discussing the properties of the current method.

Definition 3.1. Let $\widetilde{a} \in \mathcal{I F}$ be a IFN with membership and non-membership functions denoted as $\mu_{\tilde{a}}(x)$ and $\nu_{\widetilde{a}}(x)$ respectively. Let the $\alpha$-cut sets and the $\beta$-cut sets of membership and non-membership functions of $\widetilde{a}$ be $\widetilde{a}_{\alpha}=\left[L_{\widetilde{a}}^{\mu}(\alpha), R_{\tilde{a}}^{\mu}(\alpha)\right]$ and $\widetilde{a}_{\beta}=\left[L_{\widetilde{a}}^{\nu}(\beta), R_{\widetilde{a}}^{\nu}(\beta)\right]$ respectively. Then, value $\mathcal{V}_{\alpha, \beta}(\widetilde{a})$ and ambiguity $\mathcal{A}_{\alpha, \beta}(\widetilde{a})$ of $\widetilde{a}$ at decision level higher than $\alpha$ and lower than $\beta$ are defined as

$$
\begin{aligned}
& \mathcal{V}_{\alpha, \beta}(\widetilde{a})=\int_{\alpha}^{1} f(r)\left(R_{\tilde{a}}^{\mu}(r)+L_{\widetilde{a}}^{\mu}(r)\right) d r+\int_{0}^{\beta} g(r)\left(R_{\widetilde{a}}^{\nu}(r)+L_{\widetilde{a}}^{\nu}(r)\right) d r \\
& \mathcal{A}_{\alpha, \beta}(\widetilde{a})=\int_{\alpha}^{1} f(r)\left(R_{\widetilde{a}}^{\mu}(r)-L_{\widetilde{a}}^{\mu}(r)\right) d r+\int_{0}^{\beta} g(r)\left(R_{\tilde{a}}^{\nu}(r)-L_{\tilde{a}}^{\nu}(r)\right) d r
\end{aligned}
$$

where, the function $f(\alpha)$ is non-negative and non-decreasing function on the interval $[0,1]$ with $f(0)=0$ and $\int_{0}^{1} f(\alpha) d \alpha=\frac{1}{2}$; the function $g(\beta)$ is a non-negative and non-increasing function on the interval $[0,1]$ with $g(1)=0$ and $\int_{0}^{1} g(\beta) d \beta=\frac{1}{2}$. 
Proposition 3.1. Let $\widetilde{a}, \widetilde{b} \in \mathcal{I F}$ be two arbitrary IFNs, then

$$
\mathcal{V}_{\alpha, \beta}(\widetilde{a}+\widetilde{b})=\mathcal{V}_{\alpha, \beta}(\widetilde{a})+\mathcal{V}_{\alpha, \beta}(\widetilde{b})
$$

and

$$
\mathcal{V}_{\alpha, \beta}(\widetilde{a}-\widetilde{b})=\mathcal{V}_{\alpha, \beta}(\widetilde{a})-\mathcal{V}_{\alpha, \beta}(\widetilde{b})
$$

Proof. Let $\widetilde{a}, \widetilde{b} \in \mathcal{I F}$ be two IFNs with $\alpha$-cut sets and the $\beta$-cut sets of membership and non-membership functions of $\widetilde{a}, \widetilde{b}$ be $\widetilde{a}_{\alpha}=\left[L_{\widetilde{a}}^{\mu}(\alpha), R_{\widetilde{a}}^{\mu}(\alpha)\right], \widetilde{b}_{\alpha}=\left[L_{\widetilde{b}}^{\mu}(\alpha), R_{\widetilde{b}}^{\mu}(\alpha)\right]$ and $\widetilde{a}_{\beta}=$ $\left[L_{\widetilde{a}}^{\nu}(\beta), R_{\widetilde{a}}^{\nu}(\beta)\right], \widetilde{b}_{\beta}=\left[L_{\widetilde{b}}^{\nu}(\beta), R_{\widetilde{b}}^{\nu}(\beta)\right]$ respectively, then it follows that

$$
\begin{aligned}
\mathcal{V}_{\alpha, \beta}(\widetilde{a}+\widetilde{b})= & \int_{\alpha}^{1} f(r)\left[\left(R_{\widetilde{a}}^{\mu}(r)+R_{\widetilde{b}}^{\mu}(r)\right)+\left(L_{\widetilde{a}}^{\mu}(r)+L_{\widetilde{b}}^{\mu}(r)\right)\right] d r+ \\
& \int_{0}^{\beta} g(r)\left[\left(R_{\widetilde{a}}^{\nu}(r)+R_{\widetilde{b}}^{\nu}(r)\right)+\left(L_{\widetilde{a}}^{\nu}(r)+L_{\widetilde{b}}^{\nu}(r)\right)\right] d r \\
= & {\left[\int_{\alpha}^{1} f(r)\left(R_{\widetilde{a}}^{\mu}(r)+L_{\widetilde{a}}^{\mu}(r)\right) d r+\int_{0}^{\beta} g(r)\left(R_{\widetilde{a}}^{\nu}(r)+L_{\widetilde{a}}^{\nu}(r)\right) d r\right]+} \\
& {\left[\int_{\alpha}^{1} f(r)\left(R_{\widetilde{b}}^{\mu}(r)+L_{\widetilde{b}}^{\mu}(r)\right) d r+\int_{0}^{\beta} g(r)\left(R_{\widetilde{b}}^{\nu}(r)+L_{\widetilde{b}}^{\nu}(r)\right) d r\right] } \\
= & \mathcal{V}_{\alpha, \beta}(\widetilde{a})+\mathcal{V}_{\alpha, \beta}(\widetilde{b}),
\end{aligned}
$$

and

$$
\begin{aligned}
\mathcal{V}_{\alpha, \beta}(\widetilde{a}-\widetilde{b})= & \int_{\alpha}^{1} f(r)\left[\left(R_{\widetilde{a}}^{\mu}(r)-L_{\widetilde{b}}^{\mu}(r)\right)+\left(L_{\widetilde{a}}^{\mu}(r)-R_{\widetilde{b}}^{\mu}(r)\right)\right] d r+ \\
& \int_{0}^{\beta} g(r)\left[\left(R_{\widetilde{a}}^{\nu}(r)-L_{\widetilde{b}}^{\nu}(r)\right)+\left(L_{\widetilde{a}}^{\nu}(r)-R_{\widetilde{b}}^{\nu}(r)\right)\right] d r \\
= & {\left[\int_{\alpha}^{1} f(r)\left(R_{\widetilde{a}}^{\mu}(r)+L_{\widetilde{a}}^{\mu}(r)\right) d r+\int_{0}^{\beta} g(r)\left(R_{\widetilde{a}}^{\nu}(r)+L_{\widetilde{a}}^{\nu}(r)\right) d r\right]-} \\
& {\left[\int_{\alpha}^{1} f(r)\left(R_{\widetilde{b}}^{\mu}(r)+L_{\widetilde{b}}^{\mu}(r)\right) d r+\int_{0}^{\beta} g(r)\left(R_{\widetilde{b}}^{\nu}(r)+L_{\widetilde{b}}^{\nu}(r)\right) d r\right] } \\
= & \mathcal{V}_{\alpha, \beta}(\widetilde{a})-\mathcal{V}_{\alpha, \beta}(\widetilde{b}) .
\end{aligned}
$$

Proposition 3.2. Let $\widetilde{a}, \widetilde{b} \in \mathcal{I F}$ be two arbitrary IFNs, then

$$
\mathcal{A}_{\alpha, \beta}(\widetilde{a}+\widetilde{b})=\mathcal{A}_{\alpha, \beta}(\widetilde{a})+\mathcal{A}_{\alpha, \beta}(\widetilde{b}),
$$

and

$$
\mathcal{A}_{\alpha, \beta}(\widetilde{a}-\widetilde{b})=\mathcal{A}_{\alpha, \beta}(\widetilde{a})+\mathcal{A}_{\alpha, \beta}(\widetilde{b}) .
$$

Proof. Let $\widetilde{a}, \widetilde{b} \in \mathcal{I} \mathcal{F}$ be two IFNs with $\alpha$-cut sets and the $\beta$-cut sets of membership and non-membership functions of $\widetilde{a}, \widetilde{b}$ be $\widetilde{a}_{\alpha}=\left[L_{\widetilde{a}}^{\mu}(\alpha), R_{\widetilde{a}}^{\mu}(\alpha)\right], \widetilde{b}_{\alpha}=\left[L_{\widetilde{b}}^{\mu}(\alpha), R_{\widetilde{b}}^{\mu}(\alpha)\right]$ and $\widetilde{a}_{\beta}=$ 
$\left[L_{\widetilde{a}}^{\nu}(\beta), R_{\widetilde{a}}^{\nu}(\beta)\right], \widetilde{b}_{\beta}=\left[L_{\widetilde{b}}^{\nu}(\beta), R_{\widetilde{b}}^{\nu}(\beta)\right]$ respectively, then it follows that

$$
\begin{aligned}
\mathcal{A}_{\alpha, \beta}(\widetilde{a}+\widetilde{b})= & \int_{\alpha}^{1} f(r)\left[\left(R_{\widetilde{a}}^{\mu}(r)+R_{\widetilde{b}}^{\mu}(r)\right)-\left(L_{\widetilde{a}}^{\mu}(r)+L_{\widetilde{b}}^{\mu}(r)\right)\right] d r+ \\
& \int_{0}^{\beta} g(r)\left[\left(R_{\widetilde{a}}^{\nu}(r)+R_{\widetilde{b}}^{\nu}(r)\right)-\left(L_{\widetilde{a}}^{\nu}(r)+L_{\widetilde{b}}^{\nu}(r)\right)\right] d r \\
= & {\left[\int_{\alpha}^{1} f(r)\left(R_{\widetilde{a}}^{\mu}(r)-L_{\widetilde{a}}^{\mu}(r)\right) d r+\int_{0}^{\beta} g(r)\left(R_{\widetilde{a}}^{\nu}(r)-L_{\widetilde{a}}^{\nu}(r)\right) d r\right]+} \\
& {\left[\int_{\alpha}^{1} f(r)\left(R_{\widetilde{b}}^{\mu}(r)-L_{\widetilde{b}}^{\mu}(r)\right) d r+\int_{0}^{\beta} g(r)\left(R_{\widetilde{b}}^{\nu}(r)-L_{\widetilde{b}}^{\nu}(r)\right) d r\right] } \\
= & \mathcal{A}_{\alpha, \beta}(\widetilde{a})+\mathcal{A}_{\alpha, \beta}(\widetilde{b}),
\end{aligned}
$$

and

$$
\begin{aligned}
\mathcal{A}_{\alpha, \beta}(\widetilde{a}-\widetilde{b})= & \int_{\alpha}^{1} f(r)\left[\left(R_{\widetilde{a}}^{\mu}(r)-L_{\widetilde{b}}^{\mu}(r)\right)-\left(L_{\widetilde{a}}^{\mu}(r)-R_{\widetilde{b}}^{\mu}(r)\right)\right] d r+ \\
& \int_{0}^{\beta} g(r)\left[\left(R_{\widetilde{a}}^{\nu}(r)-L_{\widetilde{b}}^{\nu}(r)\right)-\left(L_{\widetilde{a}}^{\nu}(r)-R_{\widetilde{b}}^{\nu}(r)\right)\right] d r \\
= & {\left[\int_{\alpha}^{1} f(r)\left(R_{\widetilde{a}}^{\mu}(r)-L_{\widetilde{a}}^{\mu}(r)\right) d r+\int_{0}^{\beta} g(r)\left(R_{\widetilde{a}}^{\nu}(r)-L_{\widetilde{a}}^{\nu}(r)\right) d r\right]+} \\
& {\left[\int_{\alpha}^{1} f(r)\left(R_{\widetilde{b}}^{\mu}(r)-L_{\widetilde{b}}^{\mu}(r)\right) d r+\int_{0}^{\beta} g(r)\left(R_{\widetilde{b}}^{\nu}(r)-L_{\widetilde{b}}^{\nu}(r)\right) d r\right] } \\
= & \mathcal{A}_{\alpha, \beta}(\widetilde{a})+\mathcal{A}_{\alpha, \beta}(\widetilde{b}) .
\end{aligned}
$$

Proposition 3.3. Let $\widetilde{a} \in \mathcal{I F}$ be a IFN, then for any real number $k \in \mathbb{R}-\{0\}, \mathcal{V}_{\alpha, \beta}(k \widetilde{a})=$ $k \mathcal{V}_{\alpha, \beta}(\widetilde{a})$ and

$$
\mathcal{A}_{\alpha, \beta}(k \widetilde{a})= \begin{cases}k \mathcal{A}_{\alpha, \beta}(\widetilde{a}) & \text { if } k>0 \\ -k \mathcal{A}_{\alpha, \beta}(\widetilde{a}) & \text { if } k<0 .\end{cases}
$$

Proof. Let $\widetilde{a} \in \mathcal{I F}$ be a IFN with $\alpha$-cut sets and the $\beta$-cut sets of membership and nonmembership functions of $\widetilde{a}$ be $\widetilde{a}_{\alpha}=\left[L_{\widetilde{a}}^{\mu}(\alpha), R_{\widetilde{a}}^{\mu}(\alpha)\right]$ and $\widetilde{a}_{\beta}=\left[L_{\widetilde{a}}^{\nu}(\beta), R_{\widetilde{a}}^{\nu}(\beta)\right]$ respectively, then for $k(>0) \in \mathbb{R}-\{0\}$ it follows that

$$
\begin{aligned}
\mathcal{V}_{\alpha, \beta}(k \widetilde{a}) & =\int_{\alpha}^{1} f(r)\left(k R_{\widetilde{a}}^{\mu}(r)+k L_{\widetilde{a}}^{\mu}(r)\right) d r+\int_{0}^{\beta} g(r)\left(k R_{\widetilde{a}}^{\nu}(r)+k L_{\widetilde{a}}^{\nu}(r)\right) d r \\
& =k\left[\int_{\alpha}^{1} f(r)\left(R_{\widetilde{a}}^{\mu}(r)+L_{\widetilde{a}}^{\mu}(r)\right) d r+\int_{0}^{\beta} g(r)\left(R_{\widetilde{a}}^{\nu}(r)+L_{\widetilde{a}}^{\nu}(r)\right) d r\right] \\
& =k \mathcal{V}_{\alpha, \beta}(\widetilde{a}),
\end{aligned}
$$

and

$$
\begin{aligned}
\mathcal{A}_{\alpha, \beta}(k \widetilde{a}) & =\int_{\alpha}^{1} f(r)\left(k R_{\widetilde{a}}^{\mu}(r)-k L_{\widetilde{a}}^{\mu}(r)\right) d r+\int_{0}^{\beta} g(r)\left(k R_{\widetilde{a}}^{\nu}(r)-k L_{\widetilde{a}}^{\nu}(r)\right) d r \\
& =k\left[\int_{\alpha}^{1} f(r)\left(R_{\widetilde{a}}^{\mu}(r)-L_{\widetilde{a}}^{\mu}(r)\right) d r+\int_{0}^{\beta} g(r)\left(R_{\widetilde{a}}^{\nu}(r)-L_{\widetilde{a}}^{\nu}(r)\right) d r\right] \\
& =k \mathcal{A}_{\alpha, \beta}(\widetilde{a}) .
\end{aligned}
$$


Let $k<0$. Assume $k=-m<0$, then it follows that

$$
\begin{aligned}
\mathcal{V}_{\alpha, \beta}(-m \widetilde{a}) & =\int_{\alpha}^{1} f(r)\left(-m L_{\widetilde{a}}^{\mu}(r)-m R_{\widetilde{a}}^{\mu}(r)\right) d r+\int_{0}^{\beta} g(r)\left(-m L_{\widetilde{a}}^{\nu}(r)-m R_{\widetilde{a}}^{\nu}(r)\right) d r \\
& =-m\left[\int_{\alpha}^{1} f(r)\left(R_{\widetilde{a}}^{\mu}(r)+L_{\widetilde{a}}^{\mu}(r)\right) d r+\int_{0}^{\beta} g(r)\left(R_{\widetilde{a}}^{\nu}(r)+L_{\widetilde{a}}^{\nu}(r)\right) d r\right] \\
& =-m \mathcal{V}_{\alpha, \beta}(\widetilde{a}),
\end{aligned}
$$

and

$$
\begin{aligned}
\mathcal{A}_{\alpha, \beta}(-m \widetilde{a}) & =\int_{\alpha}^{1} f(r)\left(-m L_{\widetilde{a}}^{\mu}(r)+m R_{\widetilde{a}}^{\mu}(r)\right) d r+\int_{0}^{\beta} g(r)\left(-m L_{\widetilde{a}}^{\nu}(r)+m R_{\widetilde{a}}^{\nu}(r)\right) d r \\
& =m\left[\int_{\alpha}^{1} f(r)\left(R_{\widetilde{a}}^{\mu}(r)-L_{\widetilde{a}}^{\mu}(r)\right) d r+\int_{0}^{\beta} g(r)\left(R_{\widetilde{a}}^{\nu}(r)-L_{\widetilde{a}}^{\nu}(r)\right) d r\right] \\
& =m \mathcal{A}_{\alpha, \beta}(\widetilde{a}) .
\end{aligned}
$$

Proposition 3.4. Let $\widetilde{a} \in \mathcal{I F}$ be IFN and $-\widetilde{a} \in \mathcal{I F}$ be its image, then $\mathcal{V}_{\alpha, \beta}(-\widetilde{a})=-\mathcal{V}_{\alpha, \beta}(\widetilde{a})$ and $\mathcal{A}_{\alpha, \beta}(-\widetilde{a})=\mathcal{A}_{\alpha, \beta}(\widetilde{a})$.

Proof. The proof follows immediately from the Proposition 3.3, taking into account $k=-1$ in its proof.

Proposition 3.5. Let $\widetilde{a}, \widetilde{b} \in \mathcal{I F}$ be two IFNs such that $\inf \operatorname{supp}\left(\mu_{\tilde{a}}\right)>\sup \operatorname{supp}\left(\mu_{\tilde{b}}\right)$ and $\inf \operatorname{supp}\left(\nu_{\tilde{a}}\right)>\sup \operatorname{supp}\left(\nu_{\tilde{b}}\right)$, then $\mathcal{V}_{\alpha, \beta}(\widetilde{a})>\mathcal{V}_{\alpha, \beta}(\widetilde{b})$.

Proof. Let $\widetilde{a}, \widetilde{b} \in \mathcal{I F}$ with $\alpha$-cut sets and the $\beta$-cut sets of membership and non-membership functions of $\widetilde{a}, \widetilde{b}$ be $\widetilde{a}_{\alpha}=\left[L_{\tilde{a}}^{\mu}(\alpha), R_{\tilde{a}}^{\mu}(\alpha)\right], \widetilde{b}_{\alpha}=\left[L_{\tilde{b}}^{\mu}(\alpha), R_{\tilde{b}}^{\mu}(\alpha)\right]$ and $\widetilde{a}_{\beta}=\left[L_{\widetilde{a}}^{\nu}(\beta), R_{\tilde{a}}^{\nu}(\beta)\right]$, $\widetilde{b}_{\beta}=\left[L_{\tilde{b}}^{\nu}(\beta), R_{\tilde{b}}^{\nu}(\beta)\right]$ respectively. Now, if $\inf \operatorname{supp}\left(\mu_{\tilde{a}}\right)>\sup \operatorname{supp}\left(\mu_{\tilde{b}}\right)$ and $\inf \operatorname{supp}\left(\nu_{\tilde{a}}\right)>$ $\sup \operatorname{supp}\left(\nu_{\tilde{b}}\right)$, then $L_{\tilde{a}}^{\mu}(\alpha)>R_{\tilde{b}}^{\mu}(\alpha)$ and $L_{\tilde{a}}^{\nu}(\alpha)>R_{\tilde{b}}^{\nu}(\alpha)$. Thus, it implies that $R_{\tilde{a}}^{\mu}(\alpha) \geq L_{\tilde{a}}^{\mu}(\alpha)>$ $R_{\tilde{b}}^{\mu}(\alpha) \geq L_{\tilde{b}}^{\mu}(\alpha)$ and $R_{\tilde{a}}^{\nu}(\alpha) \geq L_{\tilde{a}}^{\nu}(\alpha)>R_{\tilde{b}}^{\nu}(\alpha) \geq L_{\tilde{b}}^{\nu}(\alpha)$. Hence, it follows immediately that

$$
\begin{aligned}
& R_{\tilde{a}}^{\mu}(\alpha)+L_{\tilde{a}}^{\mu}(\alpha)>R_{\tilde{b}}^{\mu}(\alpha)+L_{\tilde{b}}^{\mu}(\alpha) \\
\Longrightarrow & \int_{\alpha}^{1} f(r)\left(R_{\tilde{a}}^{\mu}(r)+L_{\tilde{a}}^{\mu}(r)\right) d r>\int_{\alpha}^{1} f(r)\left(R_{\tilde{b}}^{\mu}(r)+L_{\tilde{b}}^{\mu}(r)\right) d r
\end{aligned}
$$

and

$$
\begin{aligned}
& R_{\tilde{a}}^{\nu}(\alpha)+L_{\tilde{a}}^{\nu}(\alpha)>R_{\tilde{b}}^{\nu}(\alpha)+L_{\tilde{b}}^{\nu}(\alpha) \\
\Longrightarrow & \int_{0}^{\beta} g(r)\left(R_{\tilde{a}}^{\nu}(r)+L_{\tilde{a}}^{\nu}(r)\right) d r>\int_{0}^{\beta} g(r)\left(R_{\tilde{b}}^{\nu}(r)+L_{\tilde{b}}^{\nu}(r)\right) d r
\end{aligned}
$$

Summing the inequalities 14 and 15 , the result follows immediately.

Proposition 3.6. If $\widetilde{a} \in \mathcal{I F}$ be two IFN such that it is symmetric about the $y$-axis, then $\mathcal{V}_{\alpha, \beta}(\widetilde{a})=0$.

Proof. Let $\widetilde{a} \in \mathcal{I F}$, then the $\alpha$-cut sets and the $\beta$-cut sets of membership and non-membership functions of $\widetilde{a}$ be $\widetilde{a}_{\alpha}=\left[L_{\widetilde{a}}^{\mu}(\alpha), R_{\widetilde{a}}^{\mu}(\alpha)\right]$ and $\widetilde{a}_{\beta}=\left[L_{\widetilde{a}}^{\nu}(\beta), R_{\widetilde{a}}^{\nu}(\beta)\right]$ respectively. Since, $\widetilde{a}$ is symmetric about the $y$-axis, it follows that $-L_{\widetilde{a}}^{\mu}(\alpha)=R_{\widetilde{a}}^{\mu}(\alpha)$ and $-L_{\tilde{a}}^{\nu}(\beta)=R_{\widetilde{a}}^{\nu}(\beta)$. Thus, it is evident that $\mathcal{V}_{\alpha, \beta}(\widetilde{a})=0$. 
Proposition 3.7. For an arbitrary IFN $\widetilde{a} \in \mathcal{I F}, \mathcal{A}_{\alpha, \beta} \geq 0$. Further, $\mathcal{A}_{\alpha, \beta}=0$ if $\widetilde{a} \in \mathcal{I} \mathcal{F}$ is a crisp valued IFN.

Proof. Let $\widetilde{a} \in \mathcal{I F}$, then the $\alpha$-cut sets and the $\beta$-cut sets of membership and non-membership functions of $\widetilde{a}$ be $\widetilde{a}_{\alpha}=\left[L_{\tilde{a}}^{\mu}(\alpha), R_{\tilde{a}}^{\mu}(\alpha)\right]$ and $\widetilde{a}_{\beta}=\left[L_{\tilde{a}}^{\nu}(\beta), R_{\tilde{a}}^{\nu}(\beta)\right]$ respectively. As $R_{\tilde{a}}^{\mu}(\alpha)-L_{\tilde{a}}^{\mu}(\alpha) \geq$ 0 and $R_{\tilde{a}}^{\nu}(\alpha)-L_{\tilde{a}}^{\nu}(\alpha) \geq 0$, it follows that $\int_{\alpha}^{1} f(r)\left(R_{\tilde{a}}^{\mu}(r)-L_{\tilde{a}}^{\mu}(r)\right) d r+\int_{0}^{\beta} g(r)\left(R_{\tilde{a}}^{\nu}(r)-L_{\tilde{a}}^{\nu}(r)\right) d r \geq$ 0 . Hence, the result $\mathcal{A}_{\alpha, \beta} \geq 0$. Further, if $\widetilde{a} \in \mathcal{I F}$ is a crisp valued IFN, then immediately it follows that $\mathcal{A}_{\alpha, \beta}=0$.

Corollary 3.1. If $\widetilde{a}=\left\langle\left(a, x_{0}, y_{0}, b\right),\left(c, x_{0}, y_{0}, d\right)\right\rangle$, then for for $0 \leq \alpha<1$ and $0<\beta \leq 1$

$$
\begin{aligned}
\mathcal{V}_{\alpha, \beta}(\widetilde{a})= & \frac{1}{2}(a+b)\left(1-\alpha^{2}\right)+\frac{1}{3}\left(x_{0}+y_{0}-a-b\right)\left(1-\alpha^{3}\right)+ \\
& \left(x_{0}+y_{0}\right)\left(\beta-\beta^{2}+\frac{1}{3} \beta^{3}\right)+(c+d)\left(\frac{1}{2} \beta^{2}-\frac{1}{3} \beta^{3}\right)
\end{aligned}
$$

and

$$
\begin{aligned}
\mathcal{A}_{\alpha, \beta}(\widetilde{a})= & \frac{1}{2}(b-a)\left(1-\alpha^{2}\right)-\frac{1}{3}\left(x_{0}-y_{0}-a+b\right)\left(1-\alpha^{3}\right)+ \\
& \left(y_{0}-x_{0}\right)\left(\beta-\beta^{2}+\frac{1}{3} \beta^{3}\right)+(d-c)\left(\frac{1}{2} \beta^{2}-\frac{1}{3} \beta^{3}\right) .
\end{aligned}
$$

Proof. Let $\widetilde{a}=\left\langle\left(a, x_{0}, y_{0}, b\right),\left(c, x_{0}, y_{0}, d\right)\right\rangle$ be a IFN with membership and non-membership functions denoted as $\mu_{\tilde{a}}(x)$ and $\nu_{\tilde{a}}(x)$ as given in Eqs. 3 and 4 respectively. Let the $\alpha$-cut sets and the $\beta$-cut sets of the membership and non-membership functions of $\widetilde{a}$ be given by Eqs. 6 and 7 respectively. Choosing $f(\alpha)$ and $g(\beta)$ as $f(\alpha)=\alpha$ and $g(\beta)=1-\beta$ respectively. Then, value $\mathcal{V}_{\alpha, \beta}(\widetilde{a})$ and ambiguity $\mathcal{A}_{\alpha, \beta}(\widetilde{a})$ of $\widetilde{a}$ at decision level higher than $\alpha$ and lower than $\beta$ are obtained as

$$
\begin{aligned}
\mathcal{V}_{\alpha, \beta}(\widetilde{a})= & \int_{\alpha}^{1} f(r)\left(L_{\widetilde{a}}^{\mu}(r)+R_{\widetilde{a}}^{\mu}(r)\right) d r+\int_{0}^{\beta} g(r)\left(L_{\widetilde{a}}^{\nu}(r)+R_{\tilde{a}}^{\nu}(r)\right) d r \\
= & \int_{\alpha}^{1} r\left[a+r\left(x_{0}-a\right)+b-r\left(b-y_{0}\right)\right] d r+ \\
& \int_{0}^{\beta}(1-r)\left[x_{0}+r\left(c-x_{0}\right)+y_{0}+r\left(d-y_{0}\right)\right] d r \\
= & \frac{1}{2}(a+b)\left(1-\alpha^{2}\right)+\frac{1}{3}\left(x_{0}+y_{0}-a-b\right)\left(1-\alpha^{3}\right)+ \\
& \left(x_{0}+y_{0}\right)\left(\beta-\beta^{2}+\frac{1}{3} \beta^{3}\right)+(c+d)\left(\frac{1}{2} \beta^{2}-\frac{1}{3} \beta^{3}\right)
\end{aligned}
$$

and

$$
\begin{aligned}
\mathcal{A}_{\alpha, \beta}(\widetilde{a})= & \int_{\alpha}^{1} f(r)\left(R_{\widetilde{a}}^{\mu}(r)-L_{\widetilde{a}}^{\mu}(r)\right) d r+\int_{0}^{\beta} g(r)\left(R_{\widetilde{a}}^{\nu}(r)-L_{\widetilde{a}}^{\nu}(r)\right) d r \\
= & \int_{\alpha}^{1} r\left[b-r\left(b-y_{0}\right)-a-r\left(x_{0}-a\right)\right] d r+ \\
& \int_{0}^{\beta}(1-r)\left[y_{0}+r\left(d-y_{0}\right)-x_{0}-r\left(c-x_{0}\right)\right] d r \\
= & \frac{1}{2}(b-a)\left(1-\alpha^{2}\right)-\frac{1}{3}\left(x_{0}-y_{0}-a+b\right)\left(1-\alpha^{3}\right)+ \\
& \left(y_{0}-x_{0}\right)\left(\beta-\beta^{2}+\frac{1}{3} \beta^{3}\right)+(d-c)\left(\frac{1}{2} \beta^{2}-\frac{1}{3} \beta^{3}\right),
\end{aligned}
$$

respectively. 


\subsection{The proposed method}

In this subsection, a novel method of ranking IFNs is developed based on the notion of 'value' and 'ambiguity' of an IFN. The 'value' and 'ambiguity' of an IFN is as defined in the Definition 3.1. Although, these quantities were used in ranking IFNs, yet the method developed previously has some limitations. Hence, an attempt has been made to overcome those limitations in this current study. The proposed method is as discussed below.

Let $\widetilde{a}, \widetilde{b} \in \mathcal{I F}$ be two IFNs with $\alpha$-cut sets and the $\beta$-cut sets of membership and nonmembership functions of $\widetilde{a}, \widetilde{b}$ be $\widetilde{a}_{\alpha}=\left[L_{\widetilde{a}}^{\mu}(\alpha), R_{\widetilde{a}}^{\mu}(\alpha)\right], \widetilde{b}_{\alpha}=\left[L_{\widetilde{b}}^{\mu}(\alpha), R_{\tilde{b}}^{\mu}(\alpha)\right]$ and $\widetilde{a}_{\beta}=\left[L_{\widetilde{a}}^{\nu}(\beta), R_{\widetilde{a}}^{\nu}(\beta)\right]$, $\widetilde{b}_{\beta}=\left[L_{\widetilde{b}}^{\nu}(\beta), R_{\widetilde{b}}^{\nu}(\beta)\right]$ respectively. Let $\mathcal{V}_{\alpha, \beta}(\widetilde{a}), \mathcal{V}_{\alpha, \beta}(\widetilde{b})$ and $\mathcal{A}_{\alpha, \beta}(\widetilde{a}), \mathcal{A}_{\alpha, \beta}(\widetilde{b})$ be the values and ambiguities of $\widetilde{a}, \widetilde{b}$ respectively, at decision level higher than $\alpha(0 \leq \alpha<1)$ and lower than $\beta(0<\beta \leq 1)$ as defined in Definition 3.1. Then the ranking index, $\mathcal{R}_{\alpha, \beta}$, for decision levels higher than $\alpha(0 \leq \alpha<1)$ and lower than $\beta(0<\beta \leq 1)$ is defined as

$$
\mathcal{R}_{\alpha, \beta}(\widetilde{a}, \theta)=\mathcal{V}_{\alpha, \beta}(\widetilde{a})+\theta \mathcal{A}_{\alpha, \beta}(\widetilde{a})
$$

where $\theta: \mathcal{F} \rightarrow\{0,-1,1\}$ such that

$$
\theta= \begin{cases}0, & \text { if } \mathcal{V}_{\alpha, \beta}(\widetilde{a}) \neq \mathcal{V}_{\alpha, \beta}(\widetilde{b}) \\ -1, & \text { if } \mathcal{V}_{\alpha, \beta}(\widetilde{a})=\mathcal{V}_{\alpha, \beta}(\widetilde{b}) \text { and } t_{\theta} \geq 0 \\ 1, & \text { if } \mathcal{V}_{\alpha, \beta}(\widetilde{a})=\mathcal{V}_{\alpha, \beta}(\widetilde{b}) \text { and } t_{\theta}<0\end{cases}
$$

where $t_{\theta}=C_{\widetilde{a}}^{\mu}$ or $t_{\theta}=C_{\widetilde{a}}^{\nu}$ or $t_{\theta}=C_{\tilde{b}}^{\mu}$ or $t_{\theta}=C_{\tilde{b}}^{\nu}$ are the fuzzy centres of $\alpha$-level and $\beta$-level sets as defined in Eq. 8,

The ordering of IFNs, $\widetilde{a}, \widetilde{b} \in \mathcal{I F}$, based on the ranking index $\mathcal{R}_{\alpha, \beta}$ for decision levels higher than $\alpha(0 \leq \alpha<1)$ and lower than $\beta(0<\beta \leq 1)$ is defined by relations $\succ, \prec$ and $\sim$ as;

- $\widetilde{a} \succ \widetilde{b}$ if, and only if, $\mathcal{R}_{\alpha, \beta}(\widetilde{a}, \theta)>\mathcal{R}_{\alpha, \beta}(\widetilde{b}, \theta)$;

- $\widetilde{a} \prec \widetilde{b}$ if, and only if, $\mathcal{R}_{\alpha, \beta}(\widetilde{a}, \theta)<\mathcal{R}_{\alpha, \beta}(\widetilde{b}, \theta)$;

- $\widetilde{a} \sim \widetilde{b}$ if, and only if, $\mathcal{R}_{\alpha, \beta}(\widetilde{a}, \theta)=\mathcal{R}_{\alpha, \beta}(\widetilde{b}, \theta)$.

The order relations $\succeq$ and $\preceq$ are formulated as

- $\widetilde{a} \succeq \widetilde{b}$ if, and only if, $\widetilde{a} \succ \widetilde{b}$ or $\widetilde{a} \sim \widetilde{b}$.

- $\widetilde{a} \preceq \widetilde{b}$ if, and only if, $\widetilde{a} \prec \widetilde{b}$ or $\widetilde{a} \sim \widetilde{b}$.

Next, a few theorems related to the ranking index $\mathcal{R}_{\alpha, \beta}$ are being discussed which will be helpful in developing and discussing the properties of the current method of ordering IFNs.

Theorem 3.1. Let $\widetilde{a}, \widetilde{b} \in \mathcal{I F}$ be two arbitrary IFNs, then

$$
\mathcal{R}_{\alpha, \beta}(\widetilde{a}+\widetilde{b}, \theta)=\mathcal{R}_{\alpha, \beta}(\widetilde{a}, \theta)+\mathcal{R}_{\alpha, \beta}(\widetilde{b}, \theta) .
$$

Hence, it follows that

$$
\mathcal{R}_{\alpha, \beta}(\widetilde{a}-\widetilde{b}, \theta)=\mathcal{R}_{\alpha, \beta}(\widetilde{a}, \theta)+\mathcal{R}_{\alpha, \beta}(-\widetilde{b}, \theta) .
$$

Proof. Let $\widetilde{a}, \widetilde{b} \in \mathcal{I F}$ be two arbitrary IFNs, then it follows from the Propositions 3.1 and 3.2 that

$$
\mathcal{V}_{\alpha, \beta}(\widetilde{a}+\widetilde{b})=\mathcal{V}_{\alpha, \beta}(\widetilde{a})+\mathcal{V}_{\alpha, \beta}(\widetilde{b})
$$


and

$$
\mathcal{A}_{\alpha, \beta}(\widetilde{a}+\widetilde{b})=\mathcal{A}_{\alpha, \beta}(\widetilde{a})+\mathcal{A}_{\alpha, \beta}(\widetilde{b})
$$

Thus, the results follows as

$$
\begin{aligned}
\mathcal{R}_{\alpha, \beta}(\widetilde{a}+\widetilde{b}, \theta) & =\mathcal{V}_{\alpha, \beta}(\widetilde{a}+\widetilde{b})+\theta \mathcal{A}_{\alpha, \beta}(\widetilde{a}+\widetilde{b}) \\
& =\left(\mathcal{V}_{\alpha, \beta}(\widetilde{a})+\mathcal{V}_{\alpha, \beta}(\widetilde{b})\right)+\theta\left(\mathcal{A}_{\alpha, \beta}(\widetilde{a})+\mathcal{A}_{\alpha, \beta}(\widetilde{b})\right) \\
& =\left(\mathcal{V}_{\alpha, \beta}(\widetilde{a})+\theta \mathcal{A}_{\alpha, \beta}(\widetilde{a})\right)+\left(\mathcal{V}_{\alpha, \beta}(\widetilde{b})+\theta \mathcal{A}_{\alpha, \beta}(\widetilde{b})\right) \\
& =\mathcal{R}_{\alpha, \beta}(\widetilde{a}, \theta)+\mathcal{R}_{\alpha, \beta}(\widetilde{b}, \theta) .
\end{aligned}
$$

Eventually, it is true that $\mathcal{R}_{\alpha, \beta}(\widetilde{a}-\widetilde{b}, \theta)=\mathcal{R}_{\alpha, \beta}(\widetilde{a}+(-\widetilde{b}), \theta)=\mathcal{R}_{\alpha, \beta}(\widetilde{a}, \theta)+\mathcal{R}_{\alpha, \beta}(-\widetilde{b}, \theta)$.

Theorem 3.2. If $\widetilde{a}=a$, such that $\widetilde{a}$ is a crisp valued IFN, then $\mathcal{V}_{\alpha, \beta}(\widetilde{a})=a\left(1-\alpha^{2}\right)+$ $2 a\left[\beta-\frac{1}{2} \beta^{2}\right]$ and $\mathcal{A}_{\alpha, \beta}(\widetilde{a})=0$ for $0 \leq \alpha<1$ and $0<\beta \leq 1$.

Proof. The proof is very trivial.

Theorem 3.3. If $\widetilde{a}, \widetilde{b} \in \mathbb{R}-\{0\}$ be two crisp valued IFNs such that $\widetilde{a}=a$ and $\widetilde{b}=b$, then $\widetilde{a} \succeq \widetilde{b}$ if, and only if, $a \geq b$.

Proof. Let $a \geq b$, then $a\left(1-\alpha^{2}\right)+2 a\left[\beta-\frac{1}{2} \beta^{2}\right] \geq b\left(1-\alpha^{2}\right)+2 b\left[\beta-\frac{1}{2} \beta^{2}\right]$ for $0 \leq \alpha<1$ and $0<\beta \leq 1$. Thus, $\mathcal{V}_{\alpha, \beta}(\widetilde{a}) \geq \mathcal{V}_{\alpha, \beta}(\widetilde{b})$, which eventually leads to $\widetilde{a} \succeq \widetilde{b}$.

Conversely, let $\widetilde{a} \succeq \widetilde{b}$, then $\mathcal{V}_{\alpha, \beta}(\widetilde{a}) \geq \mathcal{V}_{\alpha, \beta}(\widetilde{b})$. Thus,

$$
a\left(1-\alpha^{2}\right)+2 a\left[\beta-\frac{1}{2} \beta^{2}\right] \geq b\left(1-\alpha^{2}\right)+2 b\left[\beta-\frac{1}{2} \beta^{2}\right]
$$

. Eventually leads to the result $a \geq b$.

Corollary 3.2. If $\widetilde{a}=0$, then $\mathcal{V}_{\alpha, \beta}(\widetilde{a})=\mathcal{A}_{\alpha, \beta}(\widetilde{a})=0$ for $0 \leq \alpha, \beta \leq 1$.

Proof. The proof is very trivial.

\subsection{Properties and validation of the proposed method}

In this subsection, the properties of the present method are stated and proved. Further, some of the reasonable properties of Wang \& Kerre [33, 34] are also stated and proved to check the rationality validation of the proposed ranking method. Further, newer properties that the present method follows are also stated and proved.

Let $\widetilde{a}, \widetilde{b}, \widetilde{c}, \widetilde{d} \in \mathcal{I F}$, then the order relation $\succeq$ satisfies the following properties.

$\mathbb{A}_{1}: \widetilde{a} \succeq \widetilde{a}$

$\mathbb{A}_{2}:$ If $\widetilde{a} \succeq \widetilde{b}$ and $\widetilde{a} \preceq \widetilde{b}$, then $\widetilde{a} \sim \widetilde{b}$.

$\mathbb{A}_{3}:$ If $\widetilde{a} \succeq \widetilde{b}$ and $\widetilde{b} \succeq \widetilde{c}$, then $\widetilde{a} \succeq \widetilde{c}$.

$\mathbb{A}_{4}:$ If $\inf \operatorname{supp}\left(\mu_{\widetilde{a}}\right)>\sup \operatorname{supp}\left(\mu_{\tilde{b}}\right)$ and $\inf \operatorname{supp}\left(\nu_{\widetilde{a}}\right)>\sup \operatorname{supp}\left(\nu_{\tilde{b}}\right)$, then $\widetilde{a} \succeq \widetilde{b}$.

$\mathbb{A}_{4}^{\prime}:$ If $\inf \operatorname{supp}\left(\mu_{\widetilde{a}}\right)>\sup \operatorname{supp}\left(\mu_{\tilde{b}}\right)$ and $\inf \operatorname{supp}\left(\nu_{\tilde{a}}\right)>\sup \operatorname{supp}\left(\nu_{\tilde{b}}\right)$, then $\widetilde{a} \succ \widetilde{b}$.

$\mathbb{A}_{5}$ : Let $\mathcal{F}$ and $\mathcal{F}$ ' be two arbitrary finite sets of fuzzy quantities in which $\mathcal{R}_{\alpha}$ can be applied and $\widetilde{a}$ and $\widetilde{b}$ are in $\mathcal{F} \cap \mathcal{F}$, then the ranking order $\widetilde{a} \succ \widetilde{b}$ by $\mathcal{R}_{\alpha}$ on $\mathcal{F}$ ' if and only if $\widetilde{a} \succ \widetilde{b}$ by $\mathcal{R}_{\alpha}$ on $\mathcal{F}$. 
$\mathbb{A}_{6}:$ If $\widetilde{a} \succeq \widetilde{b}$, then $\widetilde{a}+\widetilde{c} \succeq \widetilde{b}+\widetilde{c}$.

$\mathbb{B}_{6}:$ If $\widetilde{a}+\widetilde{c} \succeq \widetilde{b}+\widetilde{c}$, then $\widetilde{a} \succeq \widetilde{b}$.

$\mathbb{A}_{6}^{\prime}:$ If $\widetilde{a} \succ \widetilde{b}$, then $\widetilde{a}+\widetilde{c} \succ \widetilde{b}+\widetilde{c}$.

$\mathbb{B}_{6}^{\prime}:$ If $\widetilde{a}+\widetilde{c} \succ \widetilde{b}+\widetilde{c}$, then $\widetilde{a} \succ \widetilde{b}$.

$\mathbb{A}_{7}$ : Let $k \in \mathbb{R}-\{0\}$. If $\widetilde{a} \succeq \widetilde{b}$, then $k \widetilde{a} \succeq k \widetilde{b}$ if $k>0$, and $k \widetilde{a} \preceq k \widetilde{b}$ if $k<0$.

$\mathbb{B}_{7}$ : Let $k \in \mathbb{R}-\{0\}$. If $k \widetilde{a} \succeq k \widetilde{b}$, then $\widetilde{a} \succeq \widetilde{b}$ if $k>0$, and $\widetilde{a} \preceq \widetilde{b}$ if $k<0$.

$\mathbb{A}_{7}^{\prime}$ : Let $k \in \mathbb{R}-\{0\}$. If $\widetilde{a} \succ \widetilde{b}$, then $k \widetilde{a} \succ k \widetilde{b}$ if $k>0$, and $k \widetilde{a} \prec k \widetilde{b}$ if $k<0$.

$\mathbb{B}_{7}^{\prime}:$ Let $k \in \mathbb{R}-\{0\}$. If $k \widetilde{a} \succ k \widetilde{b}$, then $\widetilde{a} \succ \widetilde{b}$ if $k>0$, and $\widetilde{a} \prec \widetilde{b}$ if $k<0$.

$\mathbb{B}_{8}:$ If $\widetilde{a} \succeq \widetilde{b}$, then $\widetilde{a}-\widetilde{c} \succeq \widetilde{b}-\widetilde{c}$.

$\mathbb{B}_{8}^{\prime}$ : If $\widetilde{a} \succ \widetilde{b}$, then $\widetilde{a}-\widetilde{c} \succ \widetilde{b}-\widetilde{c}$.

$\mathbb{B}_{9}:$ If $\widetilde{a} \succeq \widetilde{b}$ and $\widetilde{c} \succeq \widetilde{d}$, then $\widetilde{a}+\widetilde{c} \succeq \widetilde{b}+\widetilde{d}$.

$\mathbb{B}_{9}^{\prime}$ : If $\widetilde{a} \succ \widetilde{b}$ and $\widetilde{c} \succ \widetilde{d}$, then $\widetilde{a}+\widetilde{c} \succ \widetilde{b}+\widetilde{d}$.

$\mathbb{B}_{10}$ : If $\widetilde{a} \succeq \widetilde{b}$, then $-\widetilde{a} \preceq-\widetilde{b}$, provided $\widetilde{a}$ and $\widetilde{b}$ are not symmetric about $y$-axis.

$\mathbb{B}_{10}^{\prime}$ : If $\widetilde{a} \succ \widetilde{b}$, then $-\widetilde{a} \prec-\widetilde{b}$, provided $\widetilde{a}$ and $\widetilde{b}$ are not symmetric about $y$-axis.

$\mathbb{B}_{11}$ : Let $\widetilde{a}, \widetilde{b} \in \mathcal{F}$ and symmetric about $y$-axis; if $\widetilde{a} \succeq \widetilde{b}$, then $-\widetilde{a} \succeq-\widetilde{b}$.

$\mathbb{B}_{11}^{\prime}$ : Let $\widetilde{a}, \widetilde{b} \in \mathcal{F}$ and symmetric about $y$-axis; if $\widetilde{a} \succ \widetilde{b}$, then $-\widetilde{a} \succ-\widetilde{b}$.

Hereafter, these reasonable properties of the proposed method are examined and proved by the theorems stated below.

Theorem 3.4. Let $\widetilde{a}, \widetilde{b}, \widetilde{c} \in \mathcal{I F}$ be arbitrary IFNs, then the relations $\succ$ and $\sim$ satisfy the axioms for the order relations given below:

(1) $\widetilde{a} \succeq \widetilde{a}$ (reflexivity).

(2) if $\widetilde{a} \succ \widetilde{b}$ and $\widetilde{b} \succ \widetilde{c}$, then $\widetilde{a} \succ \widetilde{c}$. (transitivity). The same holds for the order relation $\succeq$.

(3) $\widetilde{a} \succ \widetilde{b}$ or $\widetilde{b} \succeq \widetilde{a}$ (law of trichotomy).

(4) $\widetilde{a}=\widetilde{b}$ if and only if $\widetilde{a} \sim \widetilde{b}$.

Proof. The proof of the above statements are as follows.

(1) The proof of this statement is followed immediately.

(2) Here, $\widetilde{a} \succ \widetilde{b}$ happens, either for $\mathcal{R}_{\alpha, \beta}(\widetilde{a}, 0)>\mathcal{R}_{\alpha, \beta}(\widetilde{b}, 0)$ or $\mathcal{R}_{\alpha, \beta}(\widetilde{a}, \pm 1)>\mathcal{R}_{\alpha, \beta}(\widetilde{b}, \pm 1)$. Further, $\widetilde{b} \succ \widetilde{c}$ happens, either for $\mathcal{R}_{\alpha, \beta}(\widetilde{b}, 0)>\mathcal{R}_{\alpha, \beta}(\widetilde{c}, 0)$ or $\mathcal{R}_{\alpha, \beta}(\widetilde{b}, \pm 1)>\mathcal{R}_{\alpha, \beta}(\widetilde{c}, \pm 1)$. Taking into account these situation, the following four cases arises.

Case 1: Let $\widetilde{a} \succ \widetilde{b}$ and $\widetilde{b} \succ \widetilde{c}$ for $\mathcal{R}_{\alpha, \beta}(\widetilde{a}, 0)>\mathcal{R}_{\alpha, \beta}(\widetilde{b}, 0)$ and $\mathcal{R}_{\alpha, \beta}(\widetilde{b}, 0)>\mathcal{R}_{\alpha, \beta}(\widetilde{c}, 0)$ respectively. Eventually, $\mathcal{R}_{\alpha, \beta}(\widetilde{a}, 0)>\mathcal{R}_{\alpha, \beta}(\widetilde{c}, 0)$. Hence, the result.

Case 2: Let $\widetilde{a} \succ \widetilde{b}$ and $\widetilde{b} \succ \widetilde{c}$ for $\mathcal{R}_{\alpha, \beta}(\widetilde{a}, 0)>\mathcal{R}_{\alpha, \beta}(\widetilde{b}, 0)$ and $\mathcal{R}_{\alpha, \beta}(\widetilde{b}, \pm 1)>\mathcal{R}_{\alpha, \beta}(\widetilde{c}, \pm 1)$ respectively. Evidently, $\mathcal{V}_{\alpha, \beta}(\widetilde{a})>\mathcal{V}_{\alpha, \beta}(\widetilde{b})$ and $\mathcal{V}_{\alpha, \beta}(\widetilde{b})=\mathcal{V}_{\alpha, \beta}(\widetilde{c})$, which implies $\mathcal{V}_{\alpha, \beta}(\widetilde{a})>\mathcal{V}_{\alpha, \beta}(\widetilde{c})$. Eventually, $\mathcal{R}_{\alpha, \beta}(\widetilde{a}, 0)>\mathcal{R}_{\alpha, \beta}(\widetilde{c}, 0)$. Hence, the result.

Case 3: Let $\widetilde{a} \succ \widetilde{b}$ and $\widetilde{b} \succ \widetilde{c}$ for $\mathcal{R}_{\alpha, \beta}(\widetilde{a}, \pm 1)>\mathcal{R}_{\alpha, \beta}(\widetilde{b}, \pm 1)$ and $\mathcal{R}_{\alpha, \beta}(\widetilde{b}, \pm 0)>\mathcal{R}_{\alpha, \beta}(\widetilde{c}, 0)$ respectively. Evidently, $\mathcal{V}_{\alpha, \beta}(\widetilde{a})=\mathcal{V}_{\alpha, \beta}(\widetilde{b})$ and $\mathcal{V}_{\alpha, \beta}(\widetilde{b})>\mathcal{V}_{\alpha, \beta}(\widetilde{c})$, which implies $\mathcal{V}_{\alpha, \beta}(\widetilde{a})>\mathcal{V}_{\alpha, \beta}(\widetilde{c})$. Eventually, $\mathcal{R}_{\alpha, \beta}(\widetilde{a}, 0)>\mathcal{R}_{\alpha, \beta}(\widetilde{c}, 0)$. Hence, the result. 
Case 4: Let $\widetilde{a} \succ \widetilde{b}$ and $\widetilde{b} \succ \widetilde{c}$ for $\mathcal{R}_{\alpha, \beta}(\widetilde{a}, \pm 1)>\mathcal{R}_{\alpha, \beta}(\widetilde{b}, \pm 1)$ and $\mathcal{R}_{\alpha, \beta}(\widetilde{b}, \pm 1)>\mathcal{R}_{\alpha, \beta}(\widetilde{c}, \pm 1)$ respectively. Eventually, $\mathcal{R}_{\alpha, \beta}(\widetilde{a}, \pm 1)>\mathcal{R}_{\alpha, \beta}(\widetilde{c}, \pm 1)$. Hence, the result.

(3) This statement is followed immediately, as the order relations $\succ$ and $\sim$ particularly based on order relation $>$ and $=$ of real numbers.

(4) If $\widetilde{a}=\widetilde{b}$, then $\mathcal{R}_{\alpha, \beta}(\widetilde{a}, \theta)=\mathcal{R}_{\alpha, \beta}(\widetilde{b}, \theta)$. Thus, the statement is followed.

Theorem 3.5. Let $\widetilde{a}, \widetilde{b} \in \mathcal{I F}$ be two IFNs such that $\inf \operatorname{supp}\left(\mu_{\widetilde{a}}\right)>\sup \operatorname{supp}\left(\mu_{\tilde{b}}\right)$ and $\inf \operatorname{supp}$ $\left(\nu_{\tilde{a}}\right)>\sup \operatorname{supp}\left(\nu_{\tilde{b}}\right)$, then $\widetilde{a} \succeq \widetilde{b}$.

Proof. Let, $\inf \operatorname{supp}\left(\mu_{\tilde{a}}\right)>\sup \operatorname{supp}\left(\mu_{\tilde{b}}\right)$ and $\inf \operatorname{supp}\left(\nu_{\tilde{a}}\right)>\sup \operatorname{supp}\left(\nu_{\tilde{b}}\right)$, then by the Proposition $3.5 \mathcal{V}_{\alpha, \beta}(\widetilde{a})>\mathcal{V}_{\alpha, \beta}(\widetilde{b})$ for all $0 \leq \alpha<1$ and $0<\beta \leq 1$. Hence, $\theta=0$ which follows that $\mu \succ \nu$, in fact by definition of $\succeq, \widetilde{a} \succeq \widetilde{b}$.

Theorem 3.6. Let $\widetilde{a}, \widetilde{b} \in \mathcal{I F}$ be two IFNs such that $\inf \operatorname{supp}\left(\mu_{\tilde{a}}\right)>\sup \operatorname{supp}\left(\mu_{\tilde{b}}\right)$ and $\inf \operatorname{supp}$ $\left(\nu_{\tilde{a}}\right)>\sup \operatorname{supp}\left(\nu_{\tilde{b}}\right)$, then $\widetilde{a} \succ \widetilde{b}$.

Proof. Let, $\inf \operatorname{supp}\left(\mu_{\tilde{a}}\right)>\sup \operatorname{supp}\left(\mu_{\tilde{b}}\right)$ and $\inf \operatorname{supp}\left(\nu_{\tilde{a}}\right)>\sup \operatorname{supp}\left(\nu_{\tilde{b}}\right)$, then by the Corollary $3.5 \mathcal{V}_{\alpha, \beta}(\widetilde{a})>\mathcal{V}_{\alpha, \beta}(\widetilde{b})$ for all $0 \leq \alpha<1$ and $0<\beta \leq 1$. Hence, $\theta=0$ and $\mathcal{R}_{\alpha, \beta}(\widetilde{a}, \theta)>$ $\mathcal{R}_{\alpha, \beta}(\vec{b}, \theta)$, which eventually yields $\widetilde{a} \succ \widetilde{b}$.

Theorem 3.7. Let $\widetilde{a}, \widetilde{b}, \widetilde{c} \in \mathcal{I F}$. If $\widetilde{a} \succeq \widetilde{b}$, then $\widetilde{a}+\widetilde{c} \succeq \widetilde{b}+\widetilde{c}$.

Proof. To proof this theorem, a claim has to be made on invariance of $\theta$ in ordering $\widetilde{a}, \widetilde{b}$ and $\widetilde{a}+\widetilde{c}, \widetilde{b}+\widetilde{c}$. The claim is as follows:

Claim : The value of $\theta$ in ordering $\widetilde{a}$ and $\widetilde{b}$ is invariant in ordering $\widetilde{a}+\widetilde{c}$ and $\widetilde{b}+\widetilde{c}$. The proof of the claim is as follows: Let $\theta=0$ in ordering $\widetilde{a}$ and $\widetilde{b}$. Hence, $\mathcal{V}_{\alpha, \beta}(\widetilde{a}) \neq \mathcal{V}_{\alpha, \beta}(\widetilde{b})$, then trivially $\mathcal{V}_{\alpha, \beta}(\widetilde{a}+\widetilde{c}) \neq \mathcal{V}_{\alpha, \beta}(\widetilde{b}+\widetilde{c})$. Hence, $\theta=0$ in ordering $\widetilde{a}+\widetilde{c}$ and $\widetilde{b}+\widetilde{c}$. Let $\theta= \pm 1$ in ordering $\widetilde{a}$ and $\widetilde{b}$. Hence, $\mathcal{V}_{\alpha, \beta}(\widetilde{a})=\mathcal{V}_{\alpha, \beta}(\widetilde{b})$, then trivially $\mathcal{V}_{\alpha, \beta}(\widetilde{a}+\widetilde{c})=\mathcal{V}_{\alpha, \beta}(\widetilde{b}+\widetilde{c})$. Hence, $\theta= \pm 1$ in ordering $\widetilde{a}+\widetilde{c}$ and $\widetilde{b}+\widetilde{c}$. Hence, the claim.

Now, by the Proposition 3.1 it follows that

$$
\mathcal{R}_{\alpha, \beta}(\widetilde{a}+\widetilde{c}, \theta)=\mathcal{R}_{\alpha, \beta}(\widetilde{a}, \theta)+\mathcal{R}_{\alpha, \beta}(\widetilde{c}, \theta),
$$

and

$$
\mathcal{R}_{\alpha, \beta}(\widetilde{b}+\widetilde{c}, \theta)=\mathcal{R}_{\alpha, \beta}(\widetilde{b}, \theta)+\mathcal{R}_{\alpha, \beta}(\widetilde{c}, \theta),
$$

Hence, if $\widetilde{a} \succeq \widetilde{b}$, then it is obvious that $\mathcal{R}_{\alpha, \beta}(\widetilde{a}, \theta) \geq \mathcal{R}_{\alpha, \beta}(\widetilde{b}, \theta)$. This leads to the inequality $\mathcal{R}_{\alpha, \beta}(\widetilde{a}, \theta)+\mathcal{R}_{\alpha, \beta}(\widetilde{c}, \theta) \geq \mathcal{R}_{\alpha, \beta}(\widetilde{b}, \theta)+\mathcal{R}_{\alpha, \beta}(\widetilde{c}, \theta)$, which evidently follows the inequality $\mathcal{R}_{\alpha, \beta}(\widetilde{a}+$ $\widetilde{c}, \theta) \geq \mathcal{R}_{\alpha, \beta}(\widetilde{b}+\widetilde{c}, \theta)$. Thus, the result follows immediately.

Theorem 3.8. Let $\widetilde{a}, \widetilde{b}, \widetilde{c} \in \mathcal{I F}$. If $\widetilde{a}+\widetilde{c} \succeq \widetilde{b}+\widetilde{c}$, then $\widetilde{a} \succeq \widetilde{b}$.

Proof. Let $\widetilde{a}, \widetilde{b}, \widetilde{c} \in \mathcal{I F}$ and $\widetilde{a}+\widetilde{c} \succeq \widetilde{b}+\widetilde{c}$. Thus, it follows that $\mathcal{R}_{\alpha, \beta}(\widetilde{a}+\widetilde{c}, \theta) \geq \mathcal{R}_{\alpha, \beta}(\widetilde{b}+\widetilde{c}, \theta)$. Thus, by the Theorem 3.1, it follows that $\mathcal{R}_{\alpha, \beta}(\widetilde{a}, \theta)+\mathcal{R}_{\alpha, \beta}(\widetilde{c}, \theta) \geq \mathcal{R}_{\alpha, \beta}(\widetilde{b}, \theta)+\mathcal{R}_{\alpha, \beta}(\widetilde{c}, \theta)$. Eventually, it leads to $\mathcal{R}_{\alpha, \beta}(\widetilde{a}, \theta) \geq \mathcal{R}_{\alpha, \beta}(\widetilde{b}, \theta)$. Hence, the result follows immediately.

Theorem 3.9. Let $\widetilde{a}, \widetilde{b}, \widetilde{c} \in \mathcal{I F}$. If $\widetilde{a} \succ \widetilde{b}$, then $\widetilde{a}+\widetilde{c} \succ \widetilde{b}+\widetilde{c}$.

Proof. The proof is very trivial by taking in account ' $\succ$ ' in the proof of the Theorem 3.7 .

Theorem 3.10. Let $\widetilde{a}, \widetilde{b}, \widetilde{c} \in \mathcal{I F}$. If $\widetilde{a}+\widetilde{c} \succ \widetilde{b}+\widetilde{c}$, then $\widetilde{a} \succ \widetilde{b}$. 
Proof. The proof is very trivial by taking in account ' $\succ$ ' in the proof of the Theorem 3.8 .

Theorem 3.11. Let $\widetilde{a}, \widetilde{b} \in \mathcal{I F}$ and $k \in \mathbb{R}-\{0\}$. If $\widetilde{a} \succeq \widetilde{b}$, then $k \widetilde{a} \succeq k \widetilde{b}$ if $k>0$, and $k \widetilde{a} \preceq k \widetilde{b}$ if $k<0$.

Proof. Let $\widetilde{a} \succeq \widetilde{b}$, then $\mathcal{R}_{\alpha, \beta}(\widetilde{a}, \theta) \geq \mathcal{R}_{\alpha, \beta}(\widetilde{b}, \theta)$. Let $k>0$, then using the the Proposition 3.3 . it follows that

$$
\begin{aligned}
\mathcal{R}_{\alpha, \beta}(k \widetilde{a}, \theta) & =\mathcal{V}_{\alpha, \beta}(k \widetilde{a})+\theta \mathcal{A}_{\alpha, \beta}(k \widetilde{a}) \\
& =k\left[\mathcal{V}_{\alpha, \beta}(\widetilde{a})+\theta \mathcal{A}_{\alpha, \beta}(\widetilde{a})\right] \\
& =k \mathcal{R}_{\alpha, \beta}(\widetilde{a}, \theta) .
\end{aligned}
$$

Thus, when $\widetilde{a} \succeq \widetilde{b}$, it follows that $\mathcal{V}_{\alpha, \beta}(\widetilde{a})+\theta \mathcal{A}_{\alpha, \beta}(\widetilde{a}) \geq \mathcal{V}_{\alpha, \beta}(\widetilde{b})+\theta \mathcal{A}_{\alpha, \beta}(\widetilde{b})$. Equivalently, it follows that $k\left[\mathcal{V}_{\alpha, \beta}(\widetilde{a})+\theta \mathcal{A}_{\alpha, \beta}(\widetilde{a})\right] \geq k\left[\mathcal{V}_{\alpha, \beta}(\widetilde{b})+\theta \mathcal{A}_{\alpha, \beta}(\widetilde{b})\right]$, which can be trivially expressed as $\mathcal{V}_{\alpha, \beta}(k \widetilde{a})+\theta \mathcal{A}_{\alpha, \beta}(k \widetilde{a}) \geq \mathcal{V}_{\alpha, \beta}(k \widetilde{b})+\theta \mathcal{A}_{\alpha, \beta}(k \widetilde{b})$. Thus, the result, $k \widetilde{a} \succeq k \widetilde{b}$, follows immediately. Let $k<0$, assume $k=-m<0$, then the following cases arise.

Case 1: Let $\widetilde{a} \succeq \widetilde{b}$ for $\theta=0$, then $\mathcal{V}_{\alpha, \beta}(\widetilde{a}) \neq \mathcal{V}_{\alpha, \beta}(\widetilde{b})$. Further, $\mathcal{R}_{\alpha, \beta}(\widetilde{a}, 0) \geq \mathcal{R}_{\alpha, \beta}(\widetilde{b}, 0)$ for $\mathcal{V}_{\alpha, \beta}(\widetilde{a})+0 \cdot \mathcal{A}_{\alpha, \beta}(\widetilde{a}) \geq \mathcal{V}_{\alpha, \beta}(\widetilde{b})+0 \cdot \mathcal{A}_{\alpha, \beta}(\widetilde{b})$. Clearly, $\mathcal{V}_{\alpha, \beta}(-m \widetilde{a}) \neq \mathcal{V}_{\alpha, \beta}(-m \widetilde{b})$ and $\mathcal{V}_{\alpha, \beta}(-m \widetilde{a}) \leq \mathcal{V}_{\alpha, \beta}(-m \widetilde{b})$ as $\mathcal{V}_{\alpha, \beta}(\widetilde{a}) \geq \mathcal{V}_{\alpha, \beta}(\widetilde{b})$. Hence, $\mathcal{V}_{\alpha, \beta}(-m \widetilde{a})+0 \cdot \mathcal{A}_{\alpha, \beta}(-m \widetilde{a}) \leq$ $\mathcal{V}_{\alpha, \beta}(-m \widetilde{b})+0 \cdot \mathcal{A}_{\alpha, \beta}(-m \widetilde{b})$. Thus $\mathcal{R}_{\alpha, \beta}(-m \widetilde{a}, 0) \leq \mathcal{R}_{\alpha, \beta}(-m \widetilde{b}, 0)$. Hence, the result.

Case 2: Let $\widetilde{a} \succeq \widetilde{b}$ for $\theta= \pm 1$, then $\mathcal{V}_{\alpha, \beta}(\widetilde{a})=\mathcal{V}_{\alpha, \beta}(\widetilde{b})$. Further, $\mathcal{R}_{\alpha, \beta}(\widetilde{a}, \pm 1) \geq \mathcal{R}_{\alpha, \beta}(\widetilde{b}, \pm 1)$ for $\mathcal{V}_{\alpha, \beta}(\widetilde{a}) \pm \mathcal{A}_{\alpha, \beta}(\widetilde{a}) \geq \mathcal{V}_{\alpha, \beta}(\widetilde{b}) \pm \mathcal{A}_{\alpha, \beta}(\widetilde{b})$. Clearly, $\mathcal{V}_{\alpha, \beta}(-m \widetilde{a})=\mathcal{V}_{\alpha, \beta}(-m \widetilde{b})$ as $\mathcal{V}_{\alpha, \beta}(\widetilde{a})=$ $\mathcal{V}_{\alpha, \beta}(\widetilde{b})$. Hence, $\mathcal{V}_{\alpha, \beta}(-m \widetilde{a}) \mp \mathcal{A}_{\alpha, \beta}(-m \widetilde{a}) \leq \mathcal{V}_{\alpha, \beta}(-m \widetilde{b}) \mp \mathcal{A}_{\alpha, \beta}(-m \widetilde{b})$. Thus $\mathcal{R}_{\alpha, \beta}(-m \widetilde{a}, \mp 1) \leq$ $\mathcal{R}_{\alpha, \beta}(-m \widetilde{b}, \mp 1)$. Hence, the result.

Theorem 3.12. Let $\widetilde{a}, \widetilde{b} \in \mathcal{I F}$ and $k \in \mathbb{R}-\{0\}$. If $k \widetilde{a} \succeq k \widetilde{b}$, then $\widetilde{a} \succeq \widetilde{b}$ if $k>0$, and $\widetilde{a} \preceq \widetilde{b}$ if $k<0$.

Proof. Let $k>0$ and $k \widetilde{a} \succ k \widetilde{b}$, then $\mathcal{R}_{\alpha, \beta}(k \widetilde{a}, \theta) \geq \mathcal{R}_{\alpha, \beta}(k \widetilde{b}, \theta)$. However, by Proposition 3.3 . it follows that $k \mathcal{R}_{\alpha, \beta}(\widetilde{a}, \theta) \geq k \mathcal{R}_{\alpha, \beta}(\widetilde{b}, \theta)$. Hence, the result follows immediately. If $k<0$, let $k=-m<0$, then $-m \widetilde{a} \succeq-m \widetilde{b}$ implies that $\mathcal{R}_{\alpha, \beta}(-m \widetilde{a}, \theta) \geq \mathcal{R}_{\alpha, \beta}(-m \widetilde{b}, \theta)$. Now, the following cases arise.

Case 1: Let $-m \widetilde{a} \succeq-m \widetilde{b}$ for $\theta=0$, then $\mathcal{V}_{\alpha, \beta}(-m \widetilde{a}) \neq \mathcal{V}_{\alpha, \beta}(-m \widetilde{b})$. Further, $\mathcal{R}_{\alpha, \beta}(-m \widetilde{a}$, $0) \geq \mathcal{R}_{\alpha, \beta}(-m \widetilde{b}, 0)$ for $\mathcal{V}_{\alpha, \beta}(-m \widetilde{a})+0 \cdot \mathcal{A}_{\alpha, \beta}(-m \widetilde{a}) \geq \mathcal{V}_{\alpha, \beta}(-m \widetilde{b})+0 \cdot \mathcal{A}_{\alpha, \beta}(-m \widetilde{b})$. Clearly, $\mathcal{V}_{\alpha, \beta}(\widetilde{a}) \neq \mathcal{V}_{\alpha, \beta}(\widetilde{b})$ and $\mathcal{V}_{\alpha, \beta}(\widetilde{a}) \leq \mathcal{V}_{\alpha, \beta}(\widetilde{b})$ as $\mathcal{V}_{\alpha, \beta}(-m \widetilde{a}) \geq \mathcal{V}_{\alpha, \beta}(-m \widetilde{b})$. Hence, $\mathcal{V}_{\alpha, \beta}(\widetilde{a})+0$. $\mathcal{A}_{\alpha, \beta}(\widetilde{a}) \leq \mathcal{V}_{\alpha, \beta}(\widetilde{b})+0 \cdot \mathcal{A}_{\alpha, \beta}(\widetilde{b})$. Thus $\mathcal{R}_{\alpha, \beta}(\widetilde{a}, 0) \leq \mathcal{R}_{\alpha, \beta}(\widetilde{b}, 0)$. Hence, the result.

Case 2: Let $-m \widetilde{a} \succeq-m \widetilde{b}$ for $\theta= \pm 1$, then $\mathcal{V}_{\alpha, \beta}(-m \widetilde{a})=\mathcal{V}_{\alpha, \beta}(-m \widetilde{b})$. Further, $\mathcal{R}_{\alpha, \beta}(-m \widetilde{a}$, $\pm 1) \geq \mathcal{R}_{\alpha, \beta}(-m \widetilde{b}, \pm 1)$ for $\mathcal{V}_{\alpha, \beta}(-m \widetilde{a}) \pm \mathcal{A}_{\alpha, \beta}(-m \widetilde{a}) \geq \mathcal{V}_{\alpha, \beta}(-m \widetilde{b}) \pm \mathcal{A}_{\alpha, \beta}(-m \widetilde{b})$. Clearly, $\mathcal{V}_{\alpha, \beta}(\widetilde{a})=\mathcal{V}_{\alpha, \beta}(\widetilde{b})$ as $\mathcal{V}_{\alpha, \beta}(-m \widetilde{a})=\mathcal{V}_{\alpha, \beta}(-m \widetilde{b})$. Hence, $\mathcal{V}_{\alpha, \beta}(\widetilde{a}) \mp \mathcal{A}_{\alpha, \beta}(\widetilde{a}) \leq \mathcal{V}_{\alpha, \beta}(\widetilde{b})+$ $\mp \mathcal{A}_{\alpha, \beta}(\widetilde{b})$. Thus $\mathcal{R}_{\alpha, \beta}(\widetilde{a}, \mp 1) \leq \mathcal{R}_{\alpha, \beta}(\widetilde{b}, \mp 1)$. Hence, the result.

Theorem 3.13. Let $\widetilde{a}, \widetilde{b} \in \mathcal{I F}$ and $k \in \mathbb{R}-\{0\}$. If $\widetilde{a} \succ \widetilde{b}$, then $k \widetilde{a} \succ k \widetilde{b}$ if $k>0$, and $k \widetilde{a} \prec k \widetilde{b}$ if $k<0$.

Proof. The proof is very trivial by taking in account ' $\succ$ ' in the proof of the Theorem 3.11 .

Theorem 3.14. Let $\widetilde{a}, \widetilde{b} \in \mathcal{I F}$ and $k \in \mathbb{R}-\{0\}$. If $k \widetilde{a} \succ k \widetilde{b}$, then $\widetilde{a} \succ \widetilde{b}$ if $k>0$, and $\widetilde{a} \prec \widetilde{b}$ if $k<0$. 
Proof. The proof is very trivial by taking in account ' $\succ$ ' in the proof of the Theorem 3.12 ,

Theorem 3.15. Let $\widetilde{a}, \widetilde{b}, \widetilde{c} \in \mathcal{I F}$. If $\widetilde{a} \succeq \widetilde{b}$, then $\widetilde{a}-\widetilde{c} \succeq \widetilde{b}-\widetilde{c}$.

Proof. To proof this theorem, a claim has to be made on invariance of $\theta$ in ordering $\widetilde{a}, \widetilde{b}$ and $\widetilde{a}-\widetilde{c}, \widetilde{b}-\widetilde{c}$. The claim is as follows:

Claim : The value of $\theta$ in ordering $\widetilde{a}$ and $\widetilde{b}$ is invariant in ordering $\widetilde{a}-\widetilde{c}$ and $\widetilde{b}-\widetilde{c}$. The proof of the claim is as follows: Let $\theta=0$ in ordering $\widetilde{a}$ and $\widetilde{b}$. Hence, $\mathcal{V}_{\alpha, \beta}(\widetilde{a}) \neq \mathcal{V}_{\alpha, \beta}(\widetilde{b})$, then trivially $\mathcal{V}_{\alpha, \beta}(\widetilde{a}-\widetilde{c}) \neq \mathcal{V}_{\alpha, \beta}(\widetilde{b}-\widetilde{c})$. Hence, $\theta=0$ in ordering $\widetilde{a}-\widetilde{c}$ and $\widetilde{b}-\widetilde{c}$. Let $\theta= \pm 1$ in ordering $\widetilde{a}$ and $\widetilde{b}$. Hence, $\mathcal{V}_{\alpha, \beta}(\widetilde{a})=\mathcal{V}_{\alpha, \beta}(\widetilde{b})$, then trivially $\mathcal{V}_{\alpha, \beta}(\widetilde{a}-\widetilde{c})=\mathcal{V}_{\alpha, \beta}(\widetilde{b}-\widetilde{c})$. Hence, $\theta= \pm 1$ in ordering $\widetilde{a}-\widetilde{c}$ and $\widetilde{b}-\widetilde{c}$. Hence, the claim.

Let $\widetilde{a}, \widetilde{b}, \widetilde{c} \in \mathcal{I F}$, then by the Proposition 3.1 it follows that

$$
\mathcal{R}_{\alpha, \beta}(\widetilde{a}-\widetilde{c}, \theta)=\mathcal{R}_{\alpha, \beta}(\widetilde{a}, \theta)+\mathcal{R}_{\alpha, \beta}(-\widetilde{c}, \theta),
$$

and

$$
\mathcal{R}_{\alpha, \beta}(\widetilde{b}-\widetilde{c}, \theta)=\mathcal{R}_{\alpha, \beta}(\widetilde{b}, \theta)+\mathcal{R}_{\alpha, \beta}(-\widetilde{c}, \theta),
$$

Hence, if $\widetilde{a} \succeq \widetilde{b}$, then it is obvious that $\mathcal{R}_{\alpha, \beta}(\widetilde{a}, \theta) \geq \mathcal{R}_{\alpha, \beta}(\widetilde{b}, \theta)$. Eventually, it leads to the inequality $\mathcal{R}_{\alpha, \beta}(\widetilde{a}, \theta)+\mathcal{R}_{\alpha, \beta}(-\widetilde{c}, \theta) \geq \mathcal{R}_{\alpha, \beta}(\widetilde{b}, \theta)+\mathcal{R}_{\alpha, \beta}(-\widetilde{c}, \theta)$. Hence, evidently it follows that $\mathcal{R}_{\alpha, \beta}(\widetilde{a}+(-\widetilde{c}), \theta) \geq \mathcal{R}_{\alpha, \beta}(\widetilde{b}+(-\widetilde{c}), \theta)$. Thus, the result follows immediately.

Theorem 3.16. Let $\widetilde{a}, \widetilde{b}, \widetilde{c} \in \mathcal{I F}$. If $\widetilde{a} \succ \widetilde{b}$, then $\widetilde{a}-\widetilde{c} \succ \widetilde{b}-\widetilde{c}$.

Proof. Taking into account the proof of the Theorem 3.15 and the definition of $\succeq$, the result follows immediately.

Theorem 3.17. Let $\widetilde{a}, \widetilde{b}, \widetilde{c}, \widetilde{d} \in \mathcal{I F}$. If $\widetilde{a} \succ \widetilde{b}$ and $\widetilde{c} \succ \widetilde{d}$, then $\widetilde{a}+\widetilde{c} \succ \widetilde{b}+\widetilde{d}$.

Proof. Here, $\widetilde{a} \succ \widetilde{b}$ happens, either for $\mathcal{R}_{\alpha, \beta}(\widetilde{a}, 0)>\mathcal{R}_{\alpha, \beta}(\widetilde{b}, 0)$ or $\mathcal{R}_{\alpha, \beta}(\widetilde{a}, \pm 1)>\mathcal{R}_{\alpha, \beta}(\widetilde{b}, \pm 1)$. Further, $\widetilde{c} \succ \widetilde{d}$ happens, either for $\mathcal{R}_{\alpha, \beta}(\widetilde{c}, 0)>\mathcal{R}_{\alpha, \beta}(\widetilde{d}, 0)$ or $\mathcal{R}_{\alpha, \beta}(\widetilde{c}, \pm 1)>\mathcal{R}_{\alpha, \beta}(\widetilde{d}, \pm 1)$. Taking into account these situation, the following four cases arises.

Case 1: Let $\widetilde{a} \succ \widetilde{b}$ and $\widetilde{c} \succ \widetilde{d}$ for $\mathcal{R}_{\alpha, \beta}(\widetilde{a}, 0)>\mathcal{R}_{\alpha, \beta}(\widetilde{b}, 0)$ and $\mathcal{R}_{\alpha, \beta}(\widetilde{c}, 0)>\mathcal{R}_{\alpha, \beta}(\widetilde{d}, 0)$ respectively. Eventually, $\mathcal{R}_{\alpha, \beta}(\widetilde{a}+\widetilde{c}, 0)>\mathcal{R}_{\alpha, \beta}(\widetilde{b}+\widetilde{d}, 0)$. Hence, it follows that $\widetilde{a}+\widetilde{c} \succ \widetilde{b}+\widetilde{d}$.

Case 2: Let $\widetilde{a} \succ \widetilde{b}$ and $\widetilde{c} \succ \widetilde{d}$ for $\mathcal{R}_{\alpha, \beta}(\widetilde{a}, 0)>\mathcal{R}_{\alpha, \beta}(\widetilde{b}, 0)$ and $\mathcal{R}_{\alpha, \beta}(\widetilde{c}, \pm 1)>\mathcal{R}_{\alpha, \beta}(\widetilde{d}, \pm 1)$ respectively. Evidently, $\mathcal{V}_{\alpha, \beta}(\widetilde{a})>\mathcal{V}_{\alpha, \beta}(\widetilde{b})$ and $\mathcal{V}_{\alpha, \beta}(\widetilde{c})=\mathcal{V}_{\alpha, \beta}(\widetilde{d})$, which implies $\mathcal{V}_{\alpha, \beta}(\widetilde{a}+$ $\widetilde{c})>\mathcal{V}_{\alpha, \beta}(\widetilde{b}+\widetilde{d})$. Eventually, $\mathcal{R}_{\alpha, \beta}(\widetilde{a}+\widetilde{c}, 0)>\mathcal{R}_{\alpha, \beta}(\widetilde{b}+\widetilde{d}, 0)$. Hence, it follows that $\widetilde{a}+\widetilde{c} \succ \widetilde{b}+\widetilde{d}$.

Case 3: Let $\widetilde{a} \succ \widetilde{b}$ and $\widetilde{c} \succ \widetilde{d}$ for $\mathcal{R}_{\alpha, \beta}(\widetilde{a}, \pm 1)>\mathcal{R}_{\alpha, \beta}(\widetilde{b}, \pm 1)$ and $\mathcal{R}_{\alpha, \beta}(\widetilde{c}, \pm 0)>\mathcal{R}_{\alpha, \beta}(\widetilde{d}, 0)$ respectively. Evidently, $\mathcal{V}_{\alpha, \beta}(\widetilde{a})=\mathcal{V}_{\alpha, \beta}(\widetilde{b})$ and $\mathcal{V}_{\alpha, \beta}(\widetilde{c})>\mathcal{V}_{\alpha, \beta}(\widetilde{d})$, which implies $\mathcal{V}_{\alpha, \beta}(\widetilde{a}+$ $\widetilde{c})>\mathcal{V}_{\alpha, \beta}(\widetilde{b}+\widetilde{d})$. Eventually, $\mathcal{R}_{\alpha, \beta}(\widetilde{a}+\widetilde{c}, 0)>\mathcal{R}_{\alpha, \beta}(\widetilde{b}+\widetilde{d}, 0)$. Hence, it follows that $\widetilde{a}+\widetilde{c} \succ \widetilde{b}+\widetilde{d}$.

Case 4: Let $\widetilde{a} \succ \widetilde{b}$ and $\widetilde{c} \succ \widetilde{d}$ for $\mathcal{R}_{\alpha, \beta}(\widetilde{a}, \pm 1)>\mathcal{R}_{\alpha, \beta}(\widetilde{b}, \pm 1)$ and $\mathcal{R}_{\alpha, \beta}(\widetilde{c}, \pm 1)>\mathcal{R}_{\alpha, \beta}(\widetilde{d}, \pm 1)$ respectively. Eventually, $\mathcal{R}_{\alpha, \beta}(\widetilde{a}+\widetilde{c}, \pm 1)>\mathcal{R}_{\alpha, \beta}(\widetilde{b}+\widetilde{d}, \pm 1)$. Hence, it follows that $\widetilde{a}+\widetilde{c} \succ \widetilde{b}+\widetilde{d}$.

Theorem 3.18. Let $\widetilde{a}, \widetilde{b}, \widetilde{c}, \widetilde{d} \in \mathcal{I F}$. If $\widetilde{a} \succeq \widetilde{b}$ and $\widetilde{c} \succeq \widetilde{d}$, then $\widetilde{a}+\widetilde{c} \succeq \widetilde{b}+\widetilde{d}$. 
Proof. Taking into account the proof of the Theorem 3.17 and the definition of $\succeq$, the result follows immediately.

Theorem 3.19. Let $\widetilde{a}, \widetilde{b} \in \mathcal{I F}$ such that $\widetilde{a}$ and $\widetilde{b}$ are not symmetric about $y$-axis. If $\widetilde{a} \succeq \widetilde{b}$, then $-\widetilde{a} \preceq-\widetilde{b}$.

Proof. The proof follows immediately, taking $k=-1$ in the Theorem 3.11 .

Theorem 3.20. Let $\widetilde{a}, \widetilde{b} \in \mathcal{I F}$ such that $\widetilde{a}$ and $\widetilde{b}$ are not symmetric about $y$-axis. If $\widetilde{a} \succ \widetilde{b}$, then $-\widetilde{a} \prec-\widetilde{b}$.

Proof. Taking into account the proof of the Theorem 3.19 and the definition of $\succeq$, the result follows immediately.

Theorem 3.21. Let $\widetilde{a}, \widetilde{b} \in \mathcal{I F}$ and symmetric about $y$-axis; if $\widetilde{a} \succeq \widetilde{b}$, then $-\widetilde{a} \succeq-\widetilde{b}$.

Proof. If $\widetilde{a}, \widetilde{b} \in \mathcal{I F}$ be two symmetric IFNs about the $y$-axis, then from the Proposition 3.6 it implies $\mathcal{V}_{\alpha, \beta}(\widetilde{a})=0=\mathcal{V}_{\alpha, \beta}(\widetilde{b})$ for all $0 \leq \alpha<1$ and $0<\beta \leq 1$. Since, $\widetilde{a} \succeq \widetilde{b}$ and $t_{\theta} \geq 0$ implies $\theta=-1$. Thus, it follows that $\mathcal{R}_{\alpha, \beta}(\widetilde{a},-1) \geq \mathcal{R}_{\alpha, \beta}(\widetilde{b},-1)$. This inequality leads to the fact $-\mathcal{A}_{\alpha, \beta}(\widetilde{a}) \geq-\mathcal{A}_{\alpha, \beta}(\widetilde{b})$. Equivalently, $-\mathcal{A}_{\alpha, \beta}(-\widetilde{a}) \geq-\mathcal{A}_{\alpha, \beta}(-\widetilde{b})$. Hence, it is true that $\mathcal{R}_{\alpha, \beta}(-\widetilde{a},-1) \geq \mathcal{R}_{\alpha, \beta}(-\widetilde{b},-1)$. Hence, the result follows immediately.

Theorem 3.22. Let $\widetilde{a}, \widetilde{b} \in \mathcal{F}$ and symmetric about $y$-axis; if $\widetilde{a} \succ \widetilde{b}$, then $-\widetilde{a} \succ-\widetilde{b}$.

Proof. Taking into account the proof of the Theorem 3.21 and the definition of $\succeq$, the result follows immediately.

\section{Comparative numerical study}

In this section, a thorough comparative study is done to highlight the performance of the current method of ranking IFNs. The numerical study is conducted, considering a few sets of IFNs. The numerical study depicts the out-performance of the current method over the existing methods.

Example 4.1. Let $\widetilde{a}=\langle(1,3,3,5),(1,3,3,5)\rangle$ and $\widetilde{b}=\langle(2,3,3,4),(2,3,3,4)\rangle$ be two IFNs. The ordering of the IFNs by various methods are as depicted in Table 1. Ye [35] fails to distinguish these distinct IFNs as well as their corresponding images as the ordering of the IFNs and their corresponding images are $\widetilde{a} \sim \widetilde{b}$ and $-\widetilde{a} \sim-\widetilde{b}$ respectively. Nayagam et al. [24] ordering is illogical and depicts inconsistency in ordering the IFNs and their corresponding images as the ordering of the IFNs and their corresponding images are $\widetilde{a} \succ \widetilde{b}$ and $-\widetilde{a} \succ-\widetilde{b}$ respectively. Chutia \& Saikia [7] limitation is depicted in ordering this pair of IFNs as it fail to rank consistently the IFNs and their corresponding images which is mentioned in the same, this happens as in this case 'values' are equal and ranking is done on the quantity 'ambiguity'. The ordering of the IFNs and the corresponding images at all $(\alpha, \beta)$-levels are $\widetilde{a} \prec \widetilde{b}$ and $-\widetilde{a} \prec-\widetilde{b}$ respectively. Darehmiraki $[8$ ordering is based on the difference of the areas on the left side of the membership function and non-membership function. This method depicts indistinguishable criteria of this distinct pair of IFNs as $\widetilde{a} \sim \widetilde{b}$ and $-\widetilde{a} \sim-\widetilde{b}$ at all levels of decision-making except for the decision levels $(0.9,0.1),(0.5,0.5)$ and $(0.1,0.9)$ where it depicts indistinguishable. However, ordering of the IFNs and their corresponding images are consistent by the current method are $\widetilde{a} \prec \widetilde{b}$ and $-\widetilde{a} \succ-\widetilde{b}$ at all levels of decision-making. Hence, the current method is logical and consistent. 
Table 1: Ranking of IFNs in Examples 4.1

\begin{tabular}{lccccc}
\hline Methods & $\widetilde{a}$ & $\widetilde{b}$ & $-\widetilde{a}$ & $-\widetilde{b}$ & Decision result \\
\hline Ye [35] & 3.0000 & 3.0000 & -3.0000 & -3.0000 & $\widetilde{a} \sim \widetilde{b},-\widetilde{a} \sim-\widetilde{b}$ \\
Chutia \& Saikia [7] & & & & & \\
$(\alpha, \beta)=(0.9,0.1)$ & 0.0747 & 0.0373 & 0.0747 & 0.0373 & $\widetilde{a} \prec \widetilde{b},-\widetilde{a} \prec-\widetilde{b}$ \\
$(\alpha, \beta)=(0.5,0.5)$ & 1.3333 & 0.6667 & 1.3333 & 0.6667 & $\widetilde{a} \prec \widetilde{b},-\widetilde{a} \prec-\widetilde{b}$ \\
$\quad(\alpha, \beta)=(0.1,0.9)$ & 2.5920 & 1.2960 & 2.5920 & 1.2960 & $\widetilde{a} \prec \vec{b},-\widetilde{a} \prec-\vec{b}$ \\
Nayagam et al. [24] & 10.000 & 9.2500 & 10.000 & 9.2500 & $\widetilde{a} \succ \widetilde{b},-\widetilde{a} \succ-\widetilde{b}$ \\
$\begin{array}{c}\text { Darehmiraki [8] } \\
\quad(\alpha, \beta)=(0.9,0.1)\end{array}$ & 0.0000 & 0.0000 & 0.0000 & 0.0000 & $\widetilde{a} \sim \widetilde{b},-\widetilde{a} \sim-\widetilde{b}$ \\
$(\alpha, \beta)=(0.5,0.5)$ & 0.0000 & 0.0000 & 0.0000 & 0.0000 & $\widetilde{a} \sim \widetilde{b},-\widetilde{a} \sim-\widetilde{b}$ \\
$(\alpha, \beta)=(0.1,0.9)$ & 0.0000 & 0.0000 & 0.0000 & 0.0000 & $\widetilde{a} \sim \widetilde{b},-\widetilde{a} \sim-\widetilde{b}$ \\
Current method & & & & & \\
$(\alpha, \beta)=(0.9,0.1)$ & 0.9220 & 1.0310 & -0.9220 & -1.0310 & $\widetilde{a} \prec \widetilde{b},-\widetilde{a} \succ-\widetilde{b}$ \\
$(\alpha, \beta)=(0.5,0.5)$ & 3.2500 & 3.8750 & -3.2500 & -3.8750 & $\widetilde{a} \prec \widetilde{b},-\widetilde{a} \succ-\widetilde{b}$ \\
$(\alpha, \beta)=(0.1,0.9)$ & 3.9780 & 4.9590 & -3.9780 & -4.9590 & $\widetilde{a} \prec \widetilde{b},-\widetilde{a} \succ-\widetilde{b}$ \\
\hline
\end{tabular}

Example 4.2. Let $\widetilde{a}=\langle(1,4,4,5),(1,4,4,5)\rangle$ and $\widetilde{b}=\langle(2,3,3,6),(2,3,3,6)\rangle$ be two IFNs. The ordering of the IFNs by various methods are as depicted in Table 2. Ye 35 fails to distinguish these distinct IFNs as well as their corresponding images as the ordering of the IFNs and their corresponding images are $\widetilde{a} \sim \widetilde{b}$ and $-\widetilde{a} \sim-\widetilde{b}$ respectively. Nayagam et al. 24] ordering is illogical and depicts inconsistency in ordering the IFNs and their corresponding images as the ordering of the IFNs and their corresponding images are $\widetilde{a} \succ \widetilde{b}$ and $-\widetilde{a} \succ-\widetilde{b}$ respectively. Chutia \& Saikia [7] ordering is based on the quantity 'value', hence it rank consistently the IFNs and their corresponding images in the order $\widetilde{a} \prec \widetilde{b}$ and $-\widetilde{a} \succ-\widetilde{b}$ respectively, for all levels of decision-making. Darehmiraki 8 ordering is based on the difference of the areas of the membership function and non-membership function, hence the ordering of the IFNs is $\widetilde{a} \sim \widetilde{b}$, and their corresponding images are $-\widetilde{a} \sim-\widetilde{b}$ at decision levels $(\alpha, \beta)=(0.9,0.1),(0.5,0.5),(0.1,0.9)$. However, at other decision levels Darehmiraki [8] method depicts consistency, since it ranks the IFNs as $\widetilde{a} \prec \widetilde{b}$, and their corresponding images as $-\widetilde{a} \prec-\widetilde{b}$. Ordering of the IFNs is consistent, and efficient in ranking the IFNs and their corresponding images by the current approach and tally with the results of Darehmiraki [8] except at decision levels $(\alpha, \beta)=(0.9,0.1),(0.5,0.5),(0.1,0.9)$. Hence, the current method is logical and consistent.

Example 4.3. Consider the crisp valued IFNs $\widetilde{a}=1$ and $\widetilde{b}=2$. The ordering of the IFNs by various methods are as depicted in Table 3. The ordering by Ye [35] of the IFNs and the corresponding IFNs are $\widetilde{a} \prec \widetilde{b}$ and $-\widetilde{a} \succ-b$ which is logical. However, Nayagam et al. 24] ordering of the IFNs and the corresponding IFNs are $\widetilde{a} \prec \widetilde{b}$ and $-\widetilde{a} \prec-\widetilde{b}$ which depict inconsistency. Darehmiraki [8] preference at $(\alpha, \beta)=(0.9,0.1),(0.5,0.5)$ and $(0.1,0.9)$ for the IFNs and their corresponding images are $\widetilde{a} \sim \widetilde{b}$ and $-\widetilde{a} \sim-\widetilde{b}$ respectively. At other preference levels, this method depicts mixed and inconsistent decision. Thus, this approach fails to rank crisp-valued IFNs. Chutia \& Saikia [7] ranking is based on the 'values' calculated at various levels of $\alpha$ and $\beta$, hence this approach depicts consistency in ranking the IFNs and their corresponding images as the ordering are $\widetilde{a} \prec \widetilde{b}$ and $-\widetilde{a} \succ-\widetilde{b}$. In the current method 
Table 2: Ranking of IFNs in Examples 4.2.

\begin{tabular}{lccccc}
\hline Methods & $\widetilde{a}$ & $\widetilde{b}$ & $-\widetilde{a}$ & $-\widetilde{b}$ & Decision result \\
\hline Ye [35] & 3.5000 & 3.5000 & -3.5000 & -3.5000 & $\widetilde{a} \sim \widetilde{b},-\widetilde{a} \sim-\widetilde{b}$ \\
Chutia \& Saikia [7] & & & & & \\
$(\alpha, \beta)=(0.9,0.1)$ & 3.0027 & 2.3173 & -3.0027 & -2.3173 & $\widetilde{a} \succ \widetilde{b},-\widetilde{a} \prec-\widetilde{b}$ \\
$(\alpha, \beta)=(0.5,0.5)$ & 11.333 & 9.6667 & -11.333 & -9.6667 & $\widetilde{a} \succ \widetilde{b},-\widetilde{a} \prec-\widetilde{b}$ \\
$(\alpha, \beta)=(0.1,0.9)$ & 14.544 & 13.176 & -14.544 & -13.176 & $\widetilde{a} \succ \vec{b},-\widetilde{a} \prec-\vec{b}$ \\
Nayagam et al. [24] & -0.7500 & -3.0000 & 2.2500 & 1.0000 & $\widetilde{a} \succ \widetilde{b},-\widetilde{a} \succ-\widetilde{b}$ \\
Darehmiraki [8] & & & & & \\
$\quad(\alpha, \beta)=(0.9,0.1)$ & 0.0000 & 0.0000 & 0.0000 & 0.0000 & $\widetilde{a} \sim \widetilde{b},-\widetilde{a} \sim-\widetilde{b}$ \\
$(\alpha, \beta)=(0.5,0.5)$ & 0.0000 & 0.0000 & 0.0000 & 0.0000 & $\widetilde{a} \sim \widetilde{b},-\widetilde{a} \sim-\widetilde{b}$ \\
$(\alpha, \beta)=(0.1,0.9)$ & 0.0000 & 0.0000 & 0.0000 & 0.0000 & $\widetilde{a} \sim \widetilde{b},-\widetilde{a} \sim-\widetilde{b}$ \\
Current method & & & & & \\
$(\alpha, \beta)=(0.9,0.1)$ & 1.4110 & 1.2490 & -1.4110 & -1.2490 & $\widetilde{a} \succ \widetilde{b},-\widetilde{a} \prec-\widetilde{b}$ \\
$(\alpha, \beta)=(0.5,0.5)$ & 5.3750 & 5.1250 & -5.3750 & -5.1250 & $\widetilde{a} \succ \widetilde{b},-\widetilde{a} \prec-\widetilde{b}$ \\
$(\alpha, \beta)=(0.1,0.9)$ & 6.9390 & 6.9210 & -6.9390 & -6.9210 & $\widetilde{a} \succ \widetilde{b},-\widetilde{a} \prec-\widetilde{b}$ \\
\hline
\end{tabular}

$\mathcal{V}_{\alpha, \beta}(\widetilde{a}) \neq \mathcal{V}_{\alpha, \beta}(\widetilde{b})$, hence $\theta=0$ which suggest the decision-maker to make preference based on 'value'. Hence, the current method consistently ranks the IFNs and their corresponding IFNs by preferring $\widetilde{b}$ to $\widetilde{a}$ and $-\widetilde{a}$ to $-\widetilde{b}$ respectively.

Example 4.4. Consider the IFNs $\widetilde{a}=\langle(-1,0,0,1),(-2,0,0,2)\rangle$ and $\widetilde{a}=\langle(-2,0,0,2),(-3,0,0$, $3)\rangle$ which are symmetric about the $y$-axis. As discussed in the Theorem 3.21 and 3.22 , the ordering of the IFNs and their corresponding images are $\widetilde{a} \succ \widetilde{b}$ and $-\widetilde{a} \succ-\widetilde{b}$ respectively. The ordering by Chutia \& Saikia [7] tally with the current approach. However, Ye [35] and Darehmiraki [8] fail to distinguish this distinct pair of IFNs. Further, Nayagam et al. 24] ordering is illogical as the preference is given to the IFN $\widetilde{b}$ which has more ambiguity than $\widetilde{a}$. A comparison of the decision made by the various methods are depicted in Table 4.

\section{Discussions and conclusions}

In this paper, a novel method of ranking IFNs is being proposed based on the notion of value and ambiguity at various $(\alpha, \beta)$-levels of decision-making. The present method efficiently handles any arbitrary IFNs. Generally, in the existing methods of ranking IFNs, the images were never taken into account. In this study, the consistency in ranking the IFNs and their corresponding images, is a successful and unique attempt. Another, conclusions that can be drawn from the intuition, is that ordering of the IFNs that are symmetric about the $y$-axis and their corresponding images is same. In fact, this intuition is very clearly stated through property and discussed through the Theorems 3.21 and 3.22 . Another, attractive feature is the current method allows the decision-makers to make decision at various $(\alpha, \beta)$-levels of decision-making.

The numerical examples are also evident that the current method is superior to the existing methods, as it very efficiently handles IFNs of various types. Apart from these numerical 
Table 3: Ranking of IFNs in Examples 4.3 .

\begin{tabular}{|c|c|c|c|c|c|}
\hline Methods & $\widetilde{a}$ & $\widetilde{b}$ & $-\widetilde{a}$ & $-\widetilde{b}$ & Decision result \\
\hline Ye 35] & 1.0000 & 2.0000 & -1.0000 & -2.0000 & $\widetilde{a} \prec \widetilde{b}, \quad-\widetilde{a} \succ-\widetilde{b}$ \\
\hline \multicolumn{6}{|l|}{ Chutia \& Saikia [7] } \\
\hline$(\alpha, \beta)=(0.9,0.1)$ & 0.7600 & 1.5200 & -0.7600 & -1.5200 & $\widetilde{a} \prec \widetilde{b}, \quad-\widetilde{a} \succ-\widetilde{b}$ \\
\hline$(\alpha, \beta)=(0.5,0.5)$ & 3.0000 & 6.0000 & -3.0000 & -6.0000 & $\widetilde{a} \prec \widetilde{b}, \quad-\widetilde{a} \succ-\widetilde{b}$ \\
\hline$(\alpha, \beta)=(0.1,0.9)$ & 3.9600 & 7.9200 & -3.9600 & -7.9200 & $\widetilde{a} \prec \widetilde{b}, \quad-\widetilde{a} \succ-\widetilde{b}$ \\
\hline Nayagam et al. 24] & 1.0000 & 4.0000 & 1.0000 & 4.0000 & $\widetilde{a} \prec \widetilde{b}, \quad-\widetilde{a} \prec-\widetilde{b}$ \\
\hline \multicolumn{6}{|l|}{ Darehmiraki [8] } \\
\hline$(\alpha, \beta)=(0.9,0.1)$ & 0.0000 & 0.0000 & 0.0000 & 0.0000 & $\widetilde{a} \sim \widetilde{b}, \quad-\widetilde{a} \sim-\widetilde{b}$ \\
\hline$(\alpha, \beta)=(0.5,0.5)$ & 0.0000 & 0.0000 & 0.0000 & 0.0000 & $\widetilde{a} \sim \widetilde{b}, \quad-\widetilde{a} \sim-\widetilde{b}$ \\
\hline$(\alpha, \beta)=(0.1,0.9)$ & 0.0000 & 0.0000 & 0.0000 & 0.0000 & $\widetilde{a} \sim \widetilde{b}, \quad-\widetilde{a} \sim-\widetilde{b}$ \\
\hline \multicolumn{6}{|l|}{ Current method } \\
\hline$(\alpha, \beta)=(0.9,0.1)$ & 0.3800 & 0.7600 & -0.3800 & -0.7600 & $\widetilde{a} \prec \widetilde{b}, \quad-\widetilde{a} \succ-\widetilde{b}$ \\
\hline$(\alpha, \beta)=(0.5,0.5)$ & 1.5000 & 3.0000 & -1.5000 & -3.0000 & $\widetilde{a} \prec \widetilde{b}, \quad-\widetilde{a} \succ-\widetilde{b}$ \\
\hline$(\alpha, \beta)=(0.1,0.9)$ & 1.9800 & 3.9600 & -1.9800 & -3.9600 & $\widetilde{a} \prec \widetilde{b}, \quad-\widetilde{a} \succ-\widetilde{b}$ \\
\hline
\end{tabular}

examples, the reasonable properties of Wang \& Kerre [33] are proved under the current study. This is a very important phenomenon which depicts the novelty of the current study. Apart from these reasonable properties, newer properties are stated and proved under the current study. One of the newer property is that it discusses the consistency in ordering the IFNs and their corresponding images.

One of the limitations is that the property $\mathbb{A}_{7}$ of Wang \& Kerre [33] is not obeyed by the current method as $\mathcal{V}_{\alpha, \beta}(\widetilde{a}) \neq \mathcal{V}_{\alpha, \beta}(\widetilde{a}) \mathcal{V}_{\alpha, \beta}(\widetilde{b})$ and $\mathcal{A}_{\alpha, \beta}(\widetilde{a}) \neq \mathcal{A}_{\alpha, \beta}(\widetilde{a}) \mathcal{A}_{\alpha, \beta}(\widetilde{b})$ for IFNs $\widetilde{a}$ and $\widetilde{b}$. Hence, there is a scope for future study in this line. Another limitations is that the proposed method cannot rank more than two IFNs at a time as this approach allows pairwise comparison. Hence, to compare more than two IFNs one can use the property $\mathbb{A}_{3}$.

\section{Compliance with ethical standards}

Conflict of interest The author declare that there is no conflict of interest regarding the publication of this paper.

Ethical approval This article does not contain any studies with human participants or animals performed by the author.

Informed consent This article does not contain any studies with human participants, hence no informed consent is not declared.

\section{References}

[1] K. T. Atanassov. Intuitionistic fuzzy sets. Fuzzy Sets and Systems, 20(1):87 - 96, 1986.

[2] K. T. Atanassov. More on intuitionistic fuzzy sets. Fuzzy Sets and Systems, 33(1):37 45, 1989.

[3] K. T. Atanassov. New operations defined over the intuitionistic fuzzy sets. Fuzzy Sets and Systems, 61(2):137 - 142, 1994. 
Table 4: Ranking of IFNs in Examples 4.4 .

\begin{tabular}{|c|c|c|c|c|c|}
\hline Methods & $\widetilde{a}$ & $\widetilde{b}$ & $-\widetilde{a}$ & $-\widetilde{b}$ & Decision result \\
\hline Ye 35] & 0.0000 & 0.0000 & 0.0000 & 0.0000 & $\widetilde{a} \sim \widetilde{b}, \quad-\widetilde{a} \sim-\widetilde{b}$ \\
\hline \multicolumn{6}{|l|}{ Chutia \& Saikia [7] } \\
\hline$(\alpha, \beta)=(0.9,0.1)$ & 0.0560 & 0.0933 & 0.0560 & 0.0933 & $\widetilde{a} \succ \widetilde{b}, \quad-\widetilde{a} \succ-\widetilde{b}$ \\
\hline$(\alpha, \beta)=(0.5,0.5)$ & 1.0000 & 1.6667 & 1.0000 & 1.6667 & $\widetilde{a} \succ \underset{\sim}{\widetilde{b}}, \quad-\widetilde{a} \succ-\widetilde{b}$ \\
\hline$(\alpha, \beta)=(0.1,0.9)$ & 1.9440 & 3.2400 & 1.9440 & 3.2400 & $\widetilde{a} \succ \widetilde{b}, \quad-\widetilde{a} \succ-\widetilde{b}$ \\
\hline Nayagam et al. 24] & 0.5000 & 1.5000 & 0.5000 & 1.5000 & $\widetilde{a} \prec \widetilde{b}, \quad-\widetilde{a} \prec-\widetilde{b}$ \\
\hline \multicolumn{6}{|l|}{ Darehmiraki [8] } \\
\hline$(\alpha, \beta)=(0.9,0.1)$ & 0.0000 & 0.0000 & 0.0000 & 0.0000 & $\widetilde{a} \sim \underset{\sim}{\widetilde{b}}, \quad-\widetilde{a} \sim-\underset{\sim}{\widetilde{b}}$ \\
\hline$(\alpha, \beta)=(0.5,0.5)$ & 0.0000 & 0.0000 & 0.0000 & 0.0000 & $\widetilde{a} \sim \underset{\sim}{b}, \quad-\widetilde{a} \sim-\underset{\sim}{b}$ \\
\hline$(\alpha, \beta)=(0.1,0.9)$ & 0.0000 & 0.0000 & 0.0000 & 0.0000 & $\widetilde{a} \sim \widetilde{b}, \quad-\widetilde{a} \sim-\widetilde{b}$ \\
\hline \multicolumn{6}{|l|}{ Current method } \\
\hline$(\alpha, \beta)=(0.9,0.1)$ & -0.1183 & -0.2273 & -0.1183 & -0.2273 & $\widetilde{a} \succ \underset{\sim}{b}, \quad-\widetilde{a} \succ-\underline{\sim}$ \\
\hline$(\alpha, \beta)=(0.5,0.5)$ & -0.7917 & -1.4167 & -0.7917 & -1.4167 & $\widetilde{a} \succ \underset{\sim}{\widetilde{b}}, \quad-\widetilde{a} \succ-\underset{\sim}{\widetilde{b}}$ \\
\hline$(\alpha, \beta)=(0.1,0.9)$ & -1.3050 & -2.2860 & -1.3050 & -2.2860 & $\widetilde{a} \succ \widetilde{b}, \quad-\widetilde{a} \succ-\widetilde{b}$ \\
\hline
\end{tabular}

[4] K. T. Atanassov. Intuitionistic fuzzy sets. In Intuitionistic Fuzzy Sets, pages 1-137. Springer, 1999.

[5] K. T. Atanassov. Two theorems for intuitionistic fuzzy sets. Fuzzy Sets and Systems, 110(2):267 - 269, 2000.

[6] D. Chakraborty, D. K. Jana, and T. K. Roy. Arithmetic operations on generalized intuitionistic fuzzy number and its applications to transportation problem. OPSEARCH, $52: 431-471,2015$.

[7] R. Chutia and S. Saikia. Ranking intuitionistic fuzzy numbers at levels of decision-making and its application. Expert Systems, 35(5):e12292, 2018.

[8] M. Darehmiraki. A novel parametric ranking method for intuitionistic fuzzy numbers. Iranian Journal of Fuzzy Systems, 16(1):129-143, 2019.

[9] D. Das and P. De. Ranking of intuitionistic fuzzy numbers by new distance measure. Journal of Intelligent \&6 Fuzzy Systems, 30(2):1099-1107, 2016.

[10] S. Das and D. Guha. A centroid-based ranking method of trapezoidal intuitionistic fuzzy numbers and its application to mcdm problems. Fuzzy Information and Engineering, 8(1):41 - 74, 2016.

[11] P. K. De and D. Das. Ranking of trapezoidal intuitionistic fuzzy numbers. In $201212 t h$ International Conference on Intelligent Systems Design and Applications (ISDA), pages 184-188, Nov 2012.

[12] M. Delgado, M. Vila, and W. Voxman. On a canonical representation of fuzzy numbers. Fuzzy Sets and Systems, 93(1):125 - 135, 1998.

[13] D. Dubey and A. Mehra. Linear programming with triangular intuitionistic fuzzy number. In Proceedings of the 7th conference of the European Society for Fuzzy Logic and Technology, pages 563-569. Atlantis Press, 2011. 
[14] D. Dubois and H. Prade. Fuzzy Sets and Systems: Theory and Applications. Academic Press, Inc., Orlando, FL, USA, 1980.

[15] P. Dutta and B. Saikia. Arithmetic operations on normal semi elliptic intuitionistic fuzzy numbers and their application in decision-making. Granular Computing, 6:163-179, 2021.

[16] P. Grzegorzewski. Distances and orderings in a family of intuitionistic fuzzy numbers. In EUSFLAT Conf., pages 223-227, 2003.

[17] R. Jain. Decisionmaking in the presence of fuzzy variables. IEEE Transactions on Systems, Man and Cybernetics, SMC-6(10):698-703, Oct 1976.

[18] R. Jain. A procedure for multiple-aspect decision making using fuzzy sets. International Journal of Systems Science, 8(1):1-7, 1977.

[19] A. Kumar and M. Kaur. A ranking approach for intuitionistic fuzzy numbers and its application. Journal of Applied Research and Technology, 11(3):381 - 396, 2013.

[20] D.-F. Li. A note on "using intuitionistic fuzzy sets for fault-tree analysis on printed circuit board assembly". Microelectronics Reliability, 48(10):1741, 2008.

[21] D.-F. Li. A ratio ranking method of triangular intuitionistic fuzzy numbers and its application to MADM problems. Computers 83 Mathematics with Applications, 60(6):1557 $1570,2010$.

[22] H. B. Mitchell. Ranking-intuitionistic fuzzy numbers. International Journal of Uncertainty, Fuzziness and Knowledge-Based Systems, 12(03):377-386, 2004.

[23] J.-X. Nan, D.-F. Li, and M.-J. Zhang. A lexicographic method for matrix games with payoffs of triangular intuitionistic fuzzy numbers. International Journal of Computational Intelligence Systems, 3(3):280-289, 2010.

[24] L. G. V. Nayagam, S. Jeevaraj, and P. Dhanasekaran. A linear ordering on the class of trapezoidal intuitionistic fuzzy numbers. Expert Systems with Applications, 60:269 - 279, 2016.

[25] V. L. G. Nayagam, S. Jeevaraj, and P. Dhanasekaran. An improved ranking method for comparing trapezoidal intuitionistic fuzzy numbers and its applications to multicriteria decision making. Neural Computing \& Applications, 30:671-682, 2018.

[26] H. M. Nehi. A new ranking method for intuitionistic fuzzy numbers. International Journal of Fuzzy Systems, 12(1):80-86, 2010.

[27] S. Rezvani. Ranking method of trapezoidal intuitionistic fuzzy numbers. Annals of Fuzzy Mathematics and Informatics, 5(3):515-523, 2013.

[28] S. Salahshour, G. Shekari, and A. Hakimzadeh. A novel approach for ranking triangular intuitionistic fuzzy numbers. AWER Proedia Information Technology, 1:442-446, 2012.

[29] M. R. Seikh, P. K. Nayak, and M. Pal. Generalized triangular fuzzy numbers in intuitionistic fuzzy environ-ment. International Journal of Engineering Research and Development, 5(1):08-13, 2012.

[30] S.-P. Wan. Multi-attribute decision making method based on possibility variance coefficient of triangular intuitionistic fuzzy numbers. International Journal of Uncertainty, Fuzziness and Knowledge-Based Systems, 21(02):223-243, 2013. 
[31] S.-P. Wan and D.-F. Li. Possibility mean and variance based method for multi-attribute decision making with triangular intuitionistic fuzzy numbers. Journal of Intelligent 83 Fuzzy Systems, 24(4):743-754, 2013.

[32] J. Wang and Z. Zhang. Multi-criteria decision-making method with incomplete certain information based on intuitionistic fuzzy number. Control and decision, 24(2):226-230, 2009.

[33] X. Wang and E. E. Kerre. Reasonable properties for the ordering of fuzzy quantities (i). Fuzzy Sets and Systems, 118(3):375 - 385, 2001.

[34] X. Wang and E. E. Kerre. Reasonable properties for the ordering of fuzzy quantities (ii). Fuzzy Sets and Systems, 118(3):387 - 405, 2001.

[35] J. Ye. Expected value method for intuitionistic trapezoidal fuzzy multicriteria decisionmaking problems. Expert Systems with Applications, 38(9):11730 - 11734, 2011.

[36] X.-T. Zeng, D.-f. Li, and G.-f. Yu. A value and ambiguity-based ranking method of trapezoidal intuitionistic fuzzy numbers and application to decision making. The Scientific World Journal, 2014, 2014.

[37] X. Zhang and Z. Xu. A new method for ranking intuitionistic fuzzy values and its application in multi-attribute decision making. Fuzzy Optimization and Decision Making, 11(2):135-146, 2012. 